\section{Transport and communications}

\section{Road data}

\section{(Tables 15.4, 15.5, 15.6 and 15.7)}

The Department for Transport has undertaken significant development work over the last two years to improve its traffic estimates and measurement of traffic flow on particular stretches of the road network. This work has previously been outlined in a number of publications (Road Traffic Statistics: 2001 SB(02)23, Traffic in Great Britain Q4 2002 Data SB(03)5 and Traffic in Great Britain Q1 2003 SB(03)6).

The main point to note is that figures for 1993 to 2004 have been calculated on a different basis from years prior to 1993. Therefore, figures prior to 1993 are not directly comparable with estimates for later years. Estimates on the new basis for 1993 and subsequent years were first published by the Department on 8 May 2003 in Traffic in Great Britain Q1 2003 $\mathrm{SB}(03) 6$. A summary of the main methodological changes to take place over the last couple of years appears below.

Traffic estimates are now disaggregated for roads in urban and rural areas rather than between built-up and non builtup roads. Built-up roads were defined as those with a speed limit of $40 \mathrm{mph}$ or lower. This created difficulties in producing meaningful disaggregated traffic estimates because an increasing number of clearly rural roads were subject to a $40 \mathrm{mph}$ speed limit for safety reasons. The urban/rural split of roads is largely determined by whether roads lie within the boundaries of urban areas with a population of 10,000 or more with adjustments in some cases for major roads at the boundary.

Traffic estimates are based on the results of many 12-hour manual counts in every year, which are grossed up to estimates of annual average daily flows using expansion factors based on data from automatic traffic counters on similar roads. These averages are needed so that traffic in off-peak times, at weekends and in the summer and winter months (when only special counts are undertaken) can be taken into account when assessing the traffic at each site. For this purpose roads are now sorted into 22 groupings (previously there were only seven) and this allows a better match of manual count sites with our automatic count sites. These groupings are based on a detailed analysis of the results from all the individual automatic count sites and take into account regional groupings, road category (that is both the urban/rural classification of the road and the road class) and traffic flow levels. The groupings range from lightly-trafficked, rural minor roads in holiday areas such as Cornwall and Devon, to major roads in central London.

With the increasing interest in sub-regional statistics, we have undertaken a detailed study of traffic counts on minor roads carried out in the last ten years. This has been done in conjunction with a Geographic Information System to enable us to establish general patterns of minor road traffic in each local authority. As a result of this, we have been able to produce more reliable estimate of traffic levels in each authority in our base year of 1999. This in turn has enabled us to produce better estimates of traffic levels back to 1993, as well as more reliable estimates for 1999 onwards.

The Department created a database for major roads based on a Geographic Information System and Ordnance Survey data. This was checked by local authorities and discussed with Government Regional Offices and the Highways Agency to ensure that good local knowledge supplemented the available technical data.

\section{Road class}

(Tables 15.5 and 15.6)

Urban major and minor roads, from 1993 onwards, are defined as being within an urban area with a population of more than 10,000 people, based on the 2001 urban settlements. The definition for urban settlement can be found on the CLG web site at:

www.communities.gov.uk/planningandbuilding/ planningbuilding/planningstatistics/urbanrural.

Rural major and minor roads, from 1993 onwards, are defined as being outside an urban settlement.

\section{New vehicle registrations}

\section{(Tables 15.9)}

\section{Special concession group}

Various revisions to the vehicle taxation system were introduced on 1 July 1995 and on 29 November 1995. Separate taxation classes for farmers' goods vehicles were abolished on 1 July 1995; after this date new vehicles of this type were registered as Heavy Goods Vehicles (HGVs). The total includes 5,900 vehicles registered between 1 January and 30 June in the (now abolished) agricultural and special machines group in classes which were not eligible to register 
in the special concession group. The old agricultural and special machines taxation group was abolished at end June 1995. The group includes agricultural and mowing machines, snow ploughs and gritting vehicles. Electric vehicles are also included in this group and are no longer exempt from Vehicle Excise Duty (VED). Steam propelled vehicles were added to this group from November 1995

\section{Other licensed vehicles}

Includes three wheelers, pedestrian controlled vehicles, general haulage and showmen's tractors and recovery vehicles. Recovery vehicle tax class introduced January 1988.

\section{Special vehicles group}

The special vehicles group was created on 1 July 1995 and consists of various vehicle types over 3.5 tonnes gross weight but not required to pay VED as heavy goods vehicles. The group includes mobile cranes, work trucks, digging machines, road rollers and vehicles previously taxed as showman's goods and haulage. Figure shown for 1995 covers period from 1 July to 31 December only.

\section{National Travel Survey data}

\section{(Tables 15.1, 15.11)}

The National Travel Survey (NTS) is designed to provide a databank of personal travel information for Great Britain. It has been conducted as a continuous survey since July 1988, following ad hoc surveys since the mid-1960s. The survey is designed to identify long-term trends and is not suitable for monitoring short-term trends.

In 2006, a weighting strategy was introduced to the NTS and applied retrospectively to data back to 1995 . The weighting methodology adjusts for non-response bias and also adjusts for the drop-off in the number of trips recorded by respondents during the course of the travel week. All results now published for 1995 onwards are based on weighted data, and direct comparisons cannot be made to earlier years or previous publications.

During 2008, over 8,000 households provided details of their personal travel by filling in travel diaries over the course of a week. The drawn sample size from 2002 was nearly trebled compared with previous years following recommendations in a National Statistics Review of the NTS. This enables most results to be presented on a single year basis from 2002.

Travel included in the NTS covers all trips by British residents within Great Britain for personal reasons, including travel in the course of work. A trip is defined as a one-way course of travel having a single main purpose. It is the basic unit of personal travel defined in the survey. A round trip is split into two trips, with the first ending at a convenient point about half-way round as a notional stopping point for the outward destination and return origin. A stage is that portion of a trip defined by the use of a specific method of transport or of a specific ticket (a new stage being defined if either the mode or ticket changes). The main mode of a trip is that used for the longest stage of the trip. With stages of equal length the mode of the latest stage is used. Walks of less than 50 yards are excluded.

Travel details provided by respondents include trip purpose, method of travel, time of day and trip length. The households also provided personal information, such as their age, sex, working status, driving licence holding, and details of the cars available for their use.

Because estimates made from a sample survey depend on the particular sample chosen, they generally differ from the true values of the population. This is not usually a problem when considering large samples (such as all car trips in Great Britain), but it may give misleading information when considering data from small samples even after weighting.

The most recent editions of all NTS publications are available on the DfT website at: www.dft.gov.uk/transtat/personaltravel. Bulletins of key results are published annually. The most recent bulletin is National Travel Survey: 2008.

\section{Households with regular use of cars}

\section{(Table 15.12)}

The mid-year estimates of the percentage of households with regular use of a car or van are based on combined data from the NTS, the Expenditure and Food Survey (previously the Family Expenditure Survey) and the General Household Survey. The method for calculating these figures was changed slightly in 2006, to incorporate weighted data from the NTS and the GHS. Figures since have also been revised to incorporate weighted data. Results by area type are based on weighted data from the NTS only.

\section{Continuing Survey of Road Goods Transport}

\section{(Tables 15.3, 15.18, 15.19)}

The estimates are derived from the Continuing Survey of Road Goods Transport (CSRGT) which in 2005 was based on an average weekly returned sample of some $330 \mathrm{HGVs}$. The samples are drawn from the computerised vehicle licence records held by the Driver and Vehicle Licensing Agency. 
Questionnaires are sent to the registered keepers of the sampled vehicles asking for a description of the vehicle and its activity during the survey week. The estimates are grossed to the vehicle population, and at the overall national level have a 2 per cent margin of error (at 95 per cent confidence level). Further details and results are published in Road Freight Statistics 2005, and previously in Transport of Goods by Road in Great Britain.

\section{Methodological changes}

A key component of National Statistics outputs is a A key component of National Statistics outputs is a programme of quality reviews carried out at least every five years to ensure that such statistics are fit for purpose and that their quality and value continue to improve. A quality review of the Department for Transport's road freight surveys, including the CSRGT, was carried out in 2003. A copy of the report can be accessed at:

www.statistics.gov.uk/nsbase/methods_quality/quality_review/ downloads/NSQR30FinalReport.doc

The quality review made a number of recommendations about the CSRGT. The main methodological recommendation was that, to improve the accuracy of survey estimates, the sample strata should be amended to reflect current trends in vehicle type, weight and legislative groups. These new strata are described more fully in Appendix $C$ of the survey report. For practical and administrative reasons, changes were also made to the sample selection methodology (see Appendix B of the report). These changes have resulted in figures from 2004 not being fully comparable with those for 2003 and earlier years. Detailed comparisons should therefore be made with caution.

\section{Railways: permanent way and rolling stock}

\section{(Table 15.22)}

1. Locomotives - locos owned by Northern Ireland Railways (NIR), does not include those from the Republic of Ireland Railway System..

2. Diesel electric etc rail motor vehicles - powered passenger carrying vehicles, includes diesel electric (DE) power cars and all Construcciones y Auxiliar de Ferocarriles (CAF) vehicles. (Note: only 16 of the CAF sets were delivered to NIR at the time.)

3. Loco hauled coaches - NIR owned De Dietrich plus Gatwick but not including gen van.

4. Rail car trailers - 80 class and 450 class trailers. Not CAF, they are all powered.
5. Rolling stock for maintenance and repair - a 'standalone' figure - may or may not be included in the above totals. Anything listed as 'repair' or 'workshop' in the motive power sheets is included. Also, those CAF vehicles not yet delivered at the time.

6. The information is a 'snapshot' taken from the motive power sheets at end of March, together with any other known information.

\section{Activity at civil aerodromes}

\section{(Table 15.27)}

Figures exclude Channel Island and Isle of Man airports. Other covers local pleasure flights, scheduled service, positioning flights and non-transport charter flights for reward (for example: aerial survey work, crop dusting and delivery of empty aircraft). Non-commercial covers test and training flights, private, aeroclub, military and official flights, and business aviation, etc.

\section{Postal services and television licences}

\section{(Table 15.30)}

Letters posted category includes printed papers, newspapers, postcards and sample packets, where airmail includes letters without special charge for air transport..Business reply and freepost is now known as Response Services. 
Transport and communications

\begin{tabular}{|c|c|c|c|c|c|c|}
\hline & \multicolumn{6}{|c|}{ Males } \\
\hline & Car & Walk & $\begin{array}{r}\text { Bus } \\
\text { and coach }\end{array}$ & Rail $^{2}$ & Other ${ }^{3}$ & All modes \\
\hline Social/entertainment & 148 & 38 & 11 & 5 & 13 & 215 \\
\hline Shopping & 115 & 43 & 13 & 2 & 5 & 177 \\
\hline Other escort & 80 & 7 & 2 & 0 & 1 & 90 \\
\hline Other personal business & 64 & 21 & 6 & 1 & 4 & 96 \\
\hline Commuting & 124 & 14 & 11 & 17 & 14 & 180 \\
\hline Education & 19 & 28 & 10 & 2 & 8 & 67 \\
\hline Escort education & 16 & 8 & 0 & 0 & 0 & 25 \\
\hline Business & 30 & 2 & 1 & 3 & 1 & 37 \\
\hline Holiday/day trip & 30 & 2 & 1 & 1 & 5 & 40 \\
\hline Other, including just walk & 0 & 42 & 0 & 0 & 0 & 42 \\
\hline All purpose (=100\%) (number) & 627 & 206 & 55 & 31 & 52 & 970 \\
\hline \multicolumn{7}{|l|}{ Base } \\
\hline \multirow[t]{3}{*}{ Unweighted Base(Trips) } & 104,731 & 35,167 & 8,613 & 4,473 & 8,355 & 161,339 \\
\hline & \multicolumn{6}{|c|}{ Females } \\
\hline & Car & Walk & $\begin{array}{r}\text { Bus } \\
\text { and coach }\end{array}$ & Rail $^{2}$ & Other ${ }^{3}$ & All modes \\
\hline Social/entertainment & 159 & 37 & 13 & 5 & 9 & 223 \\
\hline Shopping & 139 & 47 & 25 & 3 & 5 & 218 \\
\hline Other escort & 85 & 12 & 3 & 0 & 1 & 101 \\
\hline Other personal business & 71 & 26 & 7 & 1 & 4 & 110 \\
\hline Communting & 87 & 19 & 14 & 9 & 5 & 133 \\
\hline Education & 19 & 23 & 9 & 1 & 5 & 57 \\
\hline Escort education & 35 & 23 & 1 & 0 & 1 & 60 \\
\hline Business & 18 & 2 & 1 & 2 & 1 & 24 \\
\hline Holiday/day trip & 33 & 2 & 2 & 1 & 4 & 42 \\
\hline Other, including just walk & 1 & 45 & 0 & 0 & 0 & 46 \\
\hline All purposes (numbers) & 646 & 236 & 75 & 23 & 34 & 1014 \\
\hline \multicolumn{7}{|l|}{ Base } \\
\hline \multirow{3}{*}{ Unweighted Base(Trips) } & 114,258 & 43,580 & 12,895 & 3,646 & 5,841 & 180,220 \\
\hline & \multicolumn{6}{|c|}{ All persons } \\
\hline & Car & Walk & $\begin{array}{r}\text { Bus } \\
\text { and coach }\end{array}$ & Rail $^{2}$ & Other $^{3}$ & All modes \\
\hline Social/entertainment & 154 & 38 & 12 & 5 & 11 & 219 \\
\hline Shopping & 127 & 45 & 19 & 2 & 5 & 198 \\
\hline Other escort & 83 & 10 & 2 & 0 & 1 & 96 \\
\hline Other personal business & 67 & 24 & 7 & 1 & 4 & 103 \\
\hline Commuting & 105 & 16 & 13 & 13 & 10 & 156 \\
\hline Education & 19 & 25 & 10 & 2 & 6 & 62 \\
\hline Escort education & 26 & 16 & 1 & 0 & 0 & 43 \\
\hline Business & 24 & 2 & 1 & 2 & 1 & 30 \\
\hline Holiday/daytrip & 32 & 2 & 2 & 1 & 4 & 41 \\
\hline Other, including just walk & 1 & 43 & 0 & 0 & 0 & 44 \\
\hline All purposes (numbers) & 637 & 221 & 65 & 27 & 42 & 992 \\
\hline \multicolumn{7}{|l|}{ Base } \\
\hline Unweighted Base(Trips) & 218,989 & 78,747 & 21,508 & 8,119 & 14,196 & 341,559 \\
\hline
\end{tabular}




\section{Transport and communications}

152 Retail Prices Index: transport components: 1998-2008

- Great Britain

Indices $(1998=100)$

\begin{tabular}{|c|c|c|c|c|c|c|c|c|}
\hline & \multirow[b]{2}{*}{ All items } & \multicolumn{5}{|c|}{ Motor vehicles } & \multirow[b]{2}{*}{ Rail fares } & \multirow[b]{2}{*}{ Bus fares } \\
\hline & & Purchase & Maintenance & $\begin{array}{r}\text { Petrol } \\
\text { and oil }\end{array}$ & $\begin{array}{r}\text { Tax and } \\
\text { insurance }\end{array}$ & $\begin{array}{r}\text { All } \\
\text { motor }^{1}\end{array}$ & & \\
\hline & ENX3 & ENX4 & ENX5 & ZCFV & ENX6 & ZCFW & ZCFX & ENX7 \\
\hline 1998 & 100.0 & 100.0 & 100.0 & 100.0 & 100.0 & 100.0 & 100.0 & 100.0 \\
\hline 1999 & 101.5 & 95.7 & 103.9 & 108.4 & 108.1 & 102.4 & 103.6 & 103.6 \\
\hline 2000 & 104.5 & 90.6 & 108.2 & 122.7 & 119.7 & 106.3 & 105.4 & 107.8 \\
\hline 2001 & 106.4 & 89.3 & 113.5 & 116.4 & 126.0 & 105.7 & 109.5 & 112.4 \\
\hline 2002 & 108.2 & 87.5 & 119.4 & 112.7 & 127.9 & 104.9 & 112.0 & 115.8 \\
\hline 2003 & 111.3 & 85.1 & 126.5 & 116.8 & 133.4 & 106.3 & 113.9 & 120.6 \\
\hline 2004 & 114.6 & 82.4 & 134.2 & 123.3 & 134.1 & 107.3 & 118.2 & 126.8 \\
\hline 2005 & 117.9 & 78.1 & 142.3 & 134.1 & 132.3 & 108.0 & 123.0 & 135.2 \\
\hline 2006 & 121.6 & 76.0 & 151.0 & 141.5 & 134.0 & 109.6 & 127.9 & 137.1 \\
\hline 2007 & 126.8 & 74.0 & 158.8 & 145.3 & 140.1 & 111.0 & 134.5 & 144.9 \\
\hline 2008 & 131.9 & 68.9 & 168.1 & 167.2 & 144.6 & 114.4 & 140.3 & 153.9 \\
\hline
\end{tabular}

1 The RPI all motor index includes purchase of a vehicle, maintenance, Source: Consumer Prices and Inflation Division, ONS: 02079444442 petrol and oil and tax and insurance. 


\begin{tabular}{|c|c|c|c|c|c|c|c|c|c|c|c|c|}
\hline & & 1998 & 1999 & 2000 & 2001 & 2002 & 2003 & 2004 & 2005 & 2006 & 2007 & 2008 \\
\hline \multicolumn{13}{|c|}{ Goods moved (billion tonnes kilometres) } \\
\hline \multicolumn{13}{|l|}{ Petroleum products } \\
\hline $\operatorname{Road}^{1}$ & ZBZP & 5.2 & 5.0 & 6.4 & 5.8 & 5.2 & 5.5 & 5.7 & 5.5 & 5.6 & 5.1 & 6.5 \\
\hline Rail $^{2}$ & ZBZQ & 1.6 & 1.5 & 1.4 & 1.2 & 1.2 & 1.2 & 1.2 & 1.2 & 1.5 & 1.6 & 1.5 \\
\hline Water $^{3}$ & ZBZR & 45.2 & 48.6 & 52.7 & 43.5 & 51.7 & 46.9 & 46.9 & 47.2 & 37.8 & 36.4 & 36.4 \\
\hline of which: coastwise & zBZS & 36.4 & 33.3 & 26.0 & 23.1 & 24.2 & 23.3 & 26.6 & 30.3 & 22.7 & 25.0 & 26.5 \\
\hline Pipeline $^{9}$ & ZBZT & 11.7 & 11.6 & 11.4 & 11.5 & 10.9 & 10.5 & 10.7 & 10.8 & 10.8 & 10.2 & 10.2 \\
\hline \multicolumn{13}{|l|}{ Coal and coke } \\
\hline Road $^{1}$ & zBZV & 2.0 & 2.2 & 1.5 & 2.1 & 1.5 & 1.5 & 1.2 & 1.5 & 1.3 & 1.6 & 1.0 \\
\hline Rail $^{2}$ & ZBZW & 4.5 & 4.8 & 4.8 & 6.2 & 5.7 & 5.8 & 6.7 & 8.3 & 8.8 & 7.7 & 7.9 \\
\hline Water $^{3}$ & zBZX & 0.5 & 0.5 & 0.2 & 0.5 & 0.3 & 0.5 & 0.3 & 0.4 & 0.5 & 0.5 & 0.5 \\
\hline All modes & ZBZY & 7.0 & 7.5 & 6.5 & 8.8 & 7.5 & 7.9 & 8.5 & 10.2 & 10.4 & 9.8 & 9.5 \\
\hline \multicolumn{13}{|l|}{ Other traffic } \\
\hline $\operatorname{Road}^{1}$ & zBZz & 153.1 & 150.5 & 151.5 & 150.6 & 152.7 & 154.7 & 155.6 & 156.4 & 159.7 & 166.4 & 156.0 \\
\hline All modes & zCAC & 175.5 & 172.0 & 178.0 & 177.4 & 179.6 & 180.0 & 180.4 & 181.9 & 185.0 & 192.2 & 179.9 \\
\hline \multicolumn{13}{|l|}{ All traffic } \\
\hline Road $^{1}$ & КСТА & 160.3 & 157.7 & 159.4 & 158.5 & 159.4 & 161.7 & 162.5 & 163.4 & 166.7 & 173.1 & 163.5 \\
\hline Rail $^{2}$ & кСтв & 17.3 & 18.2 & 18.1 & 19.4 & 18.5 & 18.9 & 20.4 & 21.7 & 21.9 & 21.2 & 20.6 \\
\hline Water $^{3}$ & $\mathrm{ZCA}$ & 56.90 & 58.70 & 67.40 & 58.80 & 67.20 & 60.90 & 59.45 & 60.87 & 51.85 & 50.80 & 49.70 \\
\hline Pipeline & КСT & 11.7 & 11.6 & 11.4 & 11.5 & 10.9 & 10.5 & 10.7 & 10.8 & 10.8 & 10.2 & 10.2 \\
\hline All modes & КСт & 246.2 & 246.2 & 256.3 & 248.2 & 256.0 & 252.0 & 253.0 & 256.8 & 251.3 & 255.3 & 244.0 \\
\hline \multicolumn{13}{|c|}{ Percentage of all traffic } \\
\hline $\operatorname{Road}^{1}$ & ZCA & 65 & 64 & 62 & 64 & 62 & 64 & 64 & 64 & 66 & 68 & 67 \\
\hline Rail $^{2}$ & ZCA & 7 & 7 & 7 & 8 & 7 & 7 & 8 & 8 & 9 & 8 & 8 \\
\hline Water $^{3}$ & ZCAG & 23 & 24 & 26 & 24 & 26 & 24 & 23 & 24 & 21 & 20 & 20 \\
\hline Pipeline & $\mathrm{ZCAH}$ & 5 & 5 & 4 & 5 & 4 & 4 & 4 & 4 & 4 & 4 & 4 \\
\hline All modes & ZCAI & 100 & 100 & 100 & 100 & 100 & 100 & 100 & 100 & 100 & 100 & 100 \\
\hline
\end{tabular}

Goods lifted (million tonnes)

Petroleum products

Road

Rail $^{2}$

Water ${ }^{3}$

of which: coastwise

Pipeline $^{9}$

All modes 4

Coal and coke

Road $^{1}$

Rail $^{2}$

Water $^{3}$

All modes

Other traffic

Road $^{1}$

Rail $^{2}$

Water $^{3}$

All modes

All traffic

Road $^{1}$

Rail $^{2}$

Water $^{3}$

Pipeline

All modes

Percentage of all traffic

Road $^{1}$

Rail $^{2}$

Water $^{3}$

Pipeline

All modes
ZCA

ZCAK

ZCA

ZCAM

ZCA

ZCAO

ZCAP

ZCAQ

ZCAR

ZCAS

ZCAT

ZCAU

ZCAW

KCTG

$\mathrm{KCTH}$

ZCAX

KCTK

$\mathrm{KCT}$

\begin{tabular}{lrr} 
ZCAY & 81 & 81 \\
ZCAZ & 5 & 5 \\
ZCBA & 7 & 7 \\
ZCBB & 7 & 8 \\
ZCBC & 100 & 100 \\
\hline
\end{tabular}

75
72
40
151
298

$\begin{array}{rr}74 & 59 \\ 60 & 67 \\ 34 & 36 \\ 151 & 146 \\ 285 & 272\end{array}$

$\begin{array}{rr}64 & 67 \\ . . & \\ 64 & 63 \\ 35 & 38 \\ 141 & 158\end{array}$

$67 \quad 70$

$\begin{array}{rrr}69 & 71 & 80 \\ . . & . . & . . \\ 57 & 56 & 58 \\ 34 & 35 & 36 \\ 159 & 146 & 147 \\ 285 & 274 & 285\end{array}$

1 All goods vehicles, including those up to 3.5 tonnes gross vehicle weight.

2 Figures for rail are for financial years (e.g 1998 will be 1998/99).

3 Figures for water are for UK traffic

4 Excludes rail.

5 See footnote 2 Table 4.4 - TSGB publication.

6 See footnote 6 Table 4.1 - TSGB publication.

7 There is a break in the series between 2003-04 and 2004-05, due to a change in the method of data collection.

8 There is a break in the series between 2006-07 and 2007-08 because coal data was not supplied by GB Railfreight prior to 2007-08.

9 Some data for 2008 is based on estmates - this survey is currently under review by DECC to improve data quality. 


\begin{tabular}{|c|c|c|c|c|c|c|c|c|c|c|c|c|}
\hline & & 1998 & 1999 & 2000 & 2001 & 2002 & 2003 & 2004 & 2005 & 2006 & 2007 & 2008 \\
\hline \multicolumn{13}{|c|}{ Billion passenger kilometres } \\
\hline \multicolumn{13}{|c|}{ Road } \\
\hline Buses and coaches & GRXK & 45 & 46 & 47 & 47 & 47 & 47 & 48 & 48 & 50 & 50 & .. \\
\hline Cars, vans and taxis & GRXG & 636 & 642 & 640 & 654 & 677 & 673 & 678 & 674 & 682 & 685 & 679 \\
\hline Motor cycles & GRXH & 4 & 5 & 5 & 5 & 5 & 6 & 6 & 6 & 6 & 6 & 6 \\
\hline Pedal cycles & GRXI & 4 & 4 & 4 & 4 & 4 & 5 & 4 & 4 & 5 & 4 & 5 \\
\hline All road & GRX & 689 & 697 & 695 & 710 & 733 & 731 & 736 & 733 & 746 & 749 & 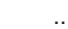 \\
\hline Rail $^{1}$ & КСT & 44 & 46 & 47 & 47 & 48 & 49 & 50 & 52 & 55 & 59 & 51 \\
\hline All modes ${ }^{2}$ & GRXM & 740 & 751 & 749 & 765 & 790 & 789 & 796 & 794 & 811 & 817 & \\
\hline
\end{tabular}

Percentages

Road

Cars, vans and taxis GRXO

Motor cycles GRXP

Pedal cycles GRXO

All road GRXR

Rail $^{1} \quad$ ZCB

Air $\quad$ zCBK

All modes ${ }^{2}$

GRXU

$\begin{array}{rr}6 & 6 \\ 86 & 86 \\ 1 & 1 \\ 1 & 1 \\ 93 & 93 \\ 6 & 6 \\ 1.0 & 1.0 \\ 100 & 100\end{array}$

$\begin{array}{rrrr}6 & 6 & 6 & 6 \\ 85 & 85 & 86 & 85 \\ 1 & 1 & 1 & 1 \\ 1 & 1 & 1 & 1 \\ 93 & 93 & 93 & 93 \\ 6 & 6 & 6 & 6 \\ 1.0 & 1.0 & 1.0 & 1.0 \\ 100 & 100 & 100 & 100\end{array}$

$\begin{array}{rrrr}6 & 6 & 6 & 6 \\ 85 & 85 & 85 & 84 \\ 1 & 1 & 1 & 1 \\ - & 1 & 1 & 1 \\ 92 & 92 & 92 & 92 \\ 6 & 7 & 7 & 7 \\ 1.0 & 1.0 & 1.0 & 1.0\end{array}$

Note: Bus and coach data not available at time of going to press and rail

$100-100$

Sources: Bus \& coach: 02079443076

data for 2008 excludes urban metros.

1 Financial years. National Rail, urban metros and modern trams

Car, m/cycle \& pedal cycle: 02079443097

Rail: 02079443076

2 Excluding travel by water

\section{Motor vehicle traffic: by road class: 1996-2006

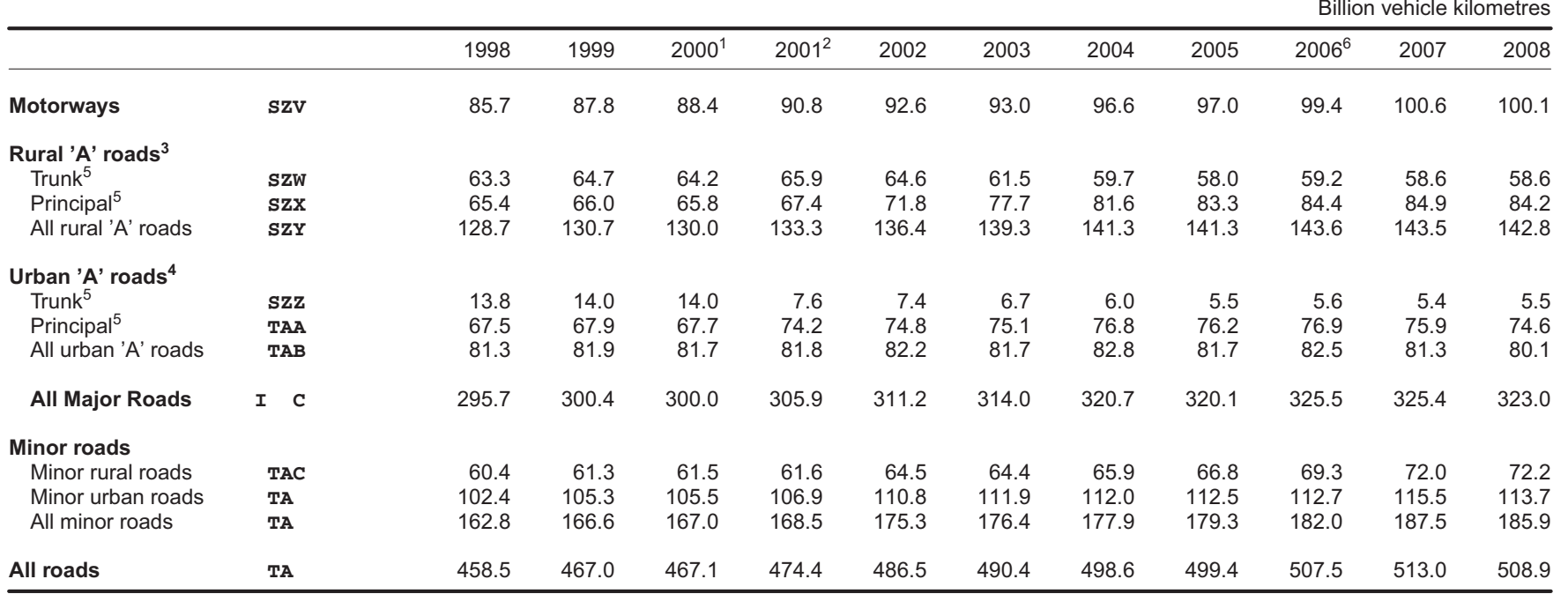

1 The decline in the use of cars and taxis in 2000 was due to the fuel dispute.

2 Figures affected by the impact of Foot and Mouth disease during 2001.

Source: Department for Transport: 02079443095

3 Rural roads; Major and minor roads, from 1993 onwards, are defined as be-

ing outside an urban area. (see definition below).

4 Urban roads; Major and minor roads, from 1993 onwards, are defined as

within an urban area with a population of 10,000 or more. These are based on the 2001 urban settlements. The definition for 'urban settlement' is in Urban and Rural area definitions: a user guide which can be found on the Department for Communities and Local Government web site at: http:/www.communities.gov.uk/publications/planningandbuilding/urbanrural

5 Figures for trunk and principal ' $A$ ' roads in England, from 2001 onwards are affected by the detrunking programme. 


\begin{tabular}{|c|c|c|c|c|c|c|c|c|c|c|c|c|}
\hline & & 1998 & 1999 & 2000 & 2001 & 2002 & 2003 & 2004 & 2005 & 2006 & 2007 & 2008 \\
\hline Trunk motorway & sz & 3376 & 3404 & 3422 & 3431 & 3433 & 3432 & 3478 & 3466 & 3503 & 3518 & 3518 \\
\hline Principal motorway & sz & 44 & 45 & 45 & 45 & 45 & 46 & 46 & 54 & 53 & 41 & 41 \\
\hline \multicolumn{13}{|l|}{ Rural 'A' roads²: } \\
\hline Trunk $^{3}$ & sz & 10585 & 10611 & 10627 & 10607 & 9973 & 9027 & 8641 & 8239 & 8277 & 8258 & 8213 \\
\hline Principal $^{3}$ & SzG & 24783 & 24852 & 24866 & 24915 & 25559 & 26498 & 26889 & 27312 & 27336 & 27346 & 27372 \\
\hline All rural 'A' roads & SZH & 35369 & 35463 & 35493 & 35522 & 35532 & 35525 & 35530 & 35550 & 35612 & 35603 & 35586 \\
\hline \multicolumn{13}{|l|}{ Urban 'A' roads ${ }^{4}$ : } \\
\hline Trunk ${ }^{3}$ & szI & 1096 & 1087 & 1074 & 762 & 705 & 587 & 506 & 444 & 446 & 425 & 420 \\
\hline Principal $^{3}$ & sz & 9931 & 10019 & 10040 & 10370 & 10436 & 10539 & 10632 & 10663 & 10696 & 10714 & 10685 \\
\hline All urban 'A' roads & szK & 11027 & 11106 & 11114 & 11132 & 11141 & 11127 & 11138 & 11107 & 11143 & 11139 & 11105 \\
\hline \multicolumn{13}{|l|}{ Minor rural roads ${ }^{5}$ : } \\
\hline$B$ roads & sz & 24586 & 24579 & 24570 & 24562 & 24554 & 24547 & 24640 & 24639 & 24574 & 24795 & 24685 \\
\hline $\mathrm{C}$ roads & SZM & 73405 & 73500 & 73593 & 73688 & 73783 & 73878 & 73363 & 73581 & 73548 & 73480 & 73582 \\
\hline Unclassified & sz & 111132 & 111350 & 111568 & 111787 & 112006 & 112231 & 109561 & 109426 & 115250 & 115365 & 115032 \\
\hline All minor rural roads & szo & 209123 & 209429 & 209731 & 210037 & 210343 & 210656 & 207565 & 207646 & 213371 & 213641 & 213299 \\
\hline \multicolumn{13}{|l|}{ Minor urban roads ${ }^{5}$ : } \\
\hline$B$ roads & SZP & 5622 & 5626 & 5630 & 5633 & 5638 & 5641 & 5538 & 5550 & 5445 & 5470 & 5476 \\
\hline $\mathrm{C}$ roads & szQ & 10986 & 11009 & 11031 & 11054 & 11076 & 11098 & 10859 & 10878 & 10921 & 10942 & 10992 \\
\hline Unclassified & SZR & 113093 & 113432 & 113772 & 114114 & 114456 & 114816 & 113520 & 113757 & 114355 & 114524 & 114450 \\
\hline All minor urban roads & szs & 129702.0 & 130068.0 & 130432.0 & 130802.0 & 131169.0 & 131556.0 & 129917.0 & 130186.0 & 130721.0 & 130936.0 & 130917.0 \\
\hline All major roads & GG $B$ & 49816 & 50018 & 50074 & 50130 & 50152 & 50130 & 50192 & 50176 & 50310 & 50302 & 50250 \\
\hline All minor roads ${ }^{5}$ & SZT & 338825 & 339496 & 340163 & 340838 & 341512 & 342212 & 337482 & 337832 & 344092 & 344577 & 344217 \\
\hline All roads & SzU & 388641 & 389515 & 390237 & 390969 & 391663 & 392342 & 387674 & 388008 & 394402 & 394879 & 394467 \\
\hline
\end{tabular}

1 A number of minor revisions have been made to the lengths of major roads 4 Urban roads: Major and minor roads, from 1993 onwards, are defined as within

from 1993 onwards.

2 Rural roads: Major and minor roads, from 1993 onwards, are defined as being outside an urban area.

3 Figures for trunk and principal 'A' roads in England, from 2001 onwards, are affected by the detrunking programme. an urban area with a population of 10,000 or more. These are based on the 2001 urban settlements. The definition for 'urban settlement' is in Urban and rural area definitions : a user guide which can be found on the Department for Communities and Local Government web site at : http//www.communities.gov.uk/publications/planningandbuilding/urbanrural

5 New information from 2004 and from 2006 has enabled better estimates of minor road lengths to be made.

Sources: National Road Traffic Survey, Department for Transport 02079443095

\section{Road traffic: by type of vehicle}

\begin{tabular}{|c|c|c|c|c|c|c|c|c|c|c|c|c|}
\hline \multirow[t]{2}{*}{ 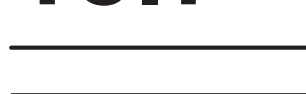 } & & \multirow[b]{2}{*}{1998} & \multirow[b]{2}{*}{1999} & \multirow[b]{2}{*}{$2000^{1}$} & \multirow[b]{2}{*}{$2001^{2}$} & \multirow[b]{2}{*}{2002} & \multirow[b]{2}{*}{2003} & \multirow[b]{2}{*}{2004} & & \multicolumn{3}{|c|}{ Billion vehicle kilometres } \\
\hline & & & & & & & & & 2005 & 2006 & $2007^{3}$ & 2008 \\
\hline Cars and taxis & TAH & 370.6 & 377.4 & 376.8 & 382.8 & 392.9 & 393.1 & 398.1 & 397.2 & 402.6 & 404.1 & 401.7 \\
\hline Motor cycles etc. & TAI & 4.1 & 4.5 & 4.6 & 4.8 & 5.1 & 5.6 & 5.2 & 5.4 & 5.2 & 5.6 & 5.1 \\
\hline Larger buses and coaches & TA & 5.2 & 5.3 & 5.2 & 5.2 & 5.2 & 5.4 & 5.2 & 5.2 & 5.4 & 5.5 & 5.2 \\
\hline Light vans ${ }^{4}$ & TAK & 50.8 & 51.6 & 52.3 & 53.7 & 55.0 & 57.9 & 60.8 & 62.6 & 65.2 & 68.4 & 68.1 \\
\hline \multicolumn{13}{|l|}{ Goods vehicles ${ }^{5}$ : } \\
\hline 2 axles rigid & TA & 11.1 & 11.6 & 11.7 & 11.5 & 11.6 & 11.7 & 11.7 & 11.5 & 11.3 & 11.1 & 10.7 \\
\hline 3 axles rigid & TAM & 1.9 & 1.7 & 1.7 & 1.8 & 1.8 & 1.8 & 1.9 & 1.9 & 1.9 & 2.0 & 2.0 \\
\hline 4 or more axles rigid & TA & 1.6 & 1.5 & 1.5 & 1.5 & 1.5 & 1.6 & 1.6 & 1.7 & 1.7 & 1.8 & 1.9 \\
\hline 3 and 4 axles artic & TAO & 3.0 & 3.0 & 2.7 & 2.5 & 2.3 & 2.2 & 2.2 & 2.0 & 1.9 & 1.8 & 1.6 \\
\hline 5 axles artic & TAP & 7.3 & 7.2 & 6.7 & 6.4 & 6.4 & 6.2 & 6.5 & 6.4 & 6.6 & 6.6 & 6.5 \\
\hline 6 or more axles artic & TAQ & 2.9 & 3.3 & 4.1 & 4.5 & 4.8 & 5.0 & 5.4 & 5.5 & 5.7 & 6.1 & 6.0 \\
\hline All & TAR & 27.7 & 28.1 & 28.2 & 28.1 & 28.3 & 28.5 & 29.4 & 29.0 & 29.1 & 29.4 & 28.7 \\
\hline Pedal cycles & URB & 4.0 & 4.1 & 4.2 & 4.2 & 4.4 & 4.5 & 4.2 & 4.4 & 4.6 & 4.2 & 4.7 \\
\hline
\end{tabular}

1 The decline in the use of cars and taxis in 2000 was due to the fuel dispute.

2 Figures affected by the impact of Foot and Mouth disease during 2001.

3 Data for 'Light vans' and 'Larger buses and coaches' for 2007 have been

4 Not exceeding $3,500 \mathrm{kgs}$ gross vehicle weight.

5 Over $3,500 \mathrm{kgs}$ gross vehicle weight. 


\begin{tabular}{|c|c|c|c|c|c|c|c|}
\hline & Petrol & Diesel & Gas/petroleum & $\begin{array}{r}\text { Gas bi-fuel/Gas } \\
\text { diesel }\end{array}$ & Hybrid-electric & Other $^{1}$ & All \\
\hline Private and light goods & 20140 & 10075 & 30 & 31 & 47 & - & 30324 \\
\hline ow: body type cars & 19959 & 6966 & 27 & 21 & 47 & - & 27021 \\
\hline $\begin{array}{l}\text { Motorcycles, scooters } \\
\text { and mopeds }\end{array}$ & 1158 & 1 & - & 0 & 0 & 1 & 1160 \\
\hline Bus & 1 & 110 & - & 0 & 0 & 0 & 111 \\
\hline Goods & 1 & 435 & - & 0 & 0 & 0 & 436 \\
\hline Special vehicles group & - & 53 & 1 & 1 & - & - & 56 \\
\hline Other non-exempt vehicles & 12 & 17 & - & 0 & 0 & 0 & 29 \\
\hline Exempt vehicles & 1322 & 727 & 2 & 1 & - & 38 & 2091 \\
\hline $\begin{array}{l}\text { OW } \\
\text { former Special concessionary group }\end{array}$ & 17 & 283 & - & 0 & 0 & 8 & 308 \\
\hline Total All Vehicles & 22634 & 11419 & 34 & 33 & 47 & 39 & 34206 \\
\hline
\end{tabular}

1 Other comprises electricity, steam, new fuel technologies, electric diesel

Source: Department for Transport: 02079443077 and fuel cells.

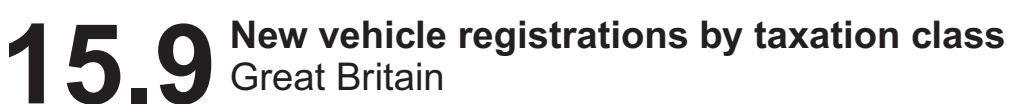

\begin{tabular}{lrrrrrrrrrrrrr} 
Thousands \\
\hline
\end{tabular}

\subsection{0}

\begin{tabular}{|c|c|c|c|c|c|c|c|c|c|c|c|c|}
\hline & & & & & & & & & & & & tages \\
\hline & & $\begin{array}{r}1989 \\
/ 90\end{array}$ & $\begin{array}{r}1991 \\
/ 92\end{array}$ & $\begin{array}{r}1998 \\
/ 99\end{array}$ & $\begin{array}{r}2001 \\
/ 02\end{array}$ & $\begin{array}{r}2002 \\
/ 03\end{array}$ & $\begin{array}{r}2003 \\
104\end{array}$ & $\begin{array}{r}2004 \\
105\end{array}$ & $\begin{array}{r}2005 \\
106\end{array}$ & $\begin{array}{r}2006 \\
107\end{array}$ & $\begin{array}{r}2007 \\
108\end{array}$ & $\begin{array}{r}2008 \\
109\end{array}$ \\
\hline Males & & & & & & & & & & & & \\
\hline Motorcycle & TRB & 72 & 69 & 69 & 67 & 66 & 67 & 66 & 66 & 67 & 68 & 68 \\
\hline Car & TRC & 58 & 57 & 51 & 47 & 47 & 46 & 46 & 46 & 46 & 47 & 49 \\
\hline Bus & TTG & - & - & 48 & 46 & 44 & 46 & 46 & 43 & 43 & 50 & 51 \\
\hline Lorry & тTн & - & - & 52 & 50 & 50 & 49 & 47 & 45 & 46 & 46 & 49 \\
\hline Females & & & & & & & & & & & & \\
\hline Motorcycle & TT & 68 & 63 & 63 & 55 & 54 & 53 & 53 & 52 & 54 & 56 & 55 \\
\hline Car & TTK & 47 & 46 & 42 & 40 & 40 & 40 & 39 & 40 & 41 & 41 & 42 \\
\hline Bus & TT & - & - & 47 & 40 & 40 & 45 & 46 & 47 & 49 & 53 & 55 \\
\hline Lorry & TTM & - & - & 50 & 47 & 46 & 48 & 45 & 45 & 47 & 48 & 52 \\
\hline All females & $T T$ & - & - & - & 41 & 40 & 40 & 40 & 40 & 41 & 42 & 42 \\
\hline Car & TTP & - & - & 46 & 43 & 43 & 43 & 42 & 42 & 43 & 44 & 45 \\
\hline Bus & TTQ & - & - & 48 & 45 & 44 & 46 & 44 & 43 & 44 & 50 & 52 \\
\hline Lorry & TTR & - & - & 52 & 56 & 49 & 49 & 46 & 45 & 46 & 46 & 49 \\
\hline All persons & TTS & - & - & - & 46 & 45 & 44 & 43 & 44 & 44 & 45 & 47 \\
\hline
\end{tabular}


Percentages and millions

Estimated

number of licence All aged $\quad 17-20 \quad$ holders

$\begin{array}{rrrrrrrr}\text { 17+ } & 17-20 & 21-29 & 30-39 & 40-49 & 50-59 & 60-69 & 70 \text { and over }\end{array}$

All adults

1975/76

$1985 / 86$

$1989 / 91$

$1992 / 94$

$1995 / 97^{2}$

$1998 / 00$

2005

2006

2007

2008

48
57
64
67
69
71

$\begin{array}{ll}28 & 59 \\ 33 & 63 \\ 43 & 72 \\ 48 & 75 \\ 43 & 74 \\ 41 & 75\end{array}$

$\begin{array}{ll}67 & 60 \\ 74 & 71 \\ 77 & 78 \\ 82 & 79 \\ 81 & 81 \\ 84 & 83\end{array}$

$\begin{array}{ll}50 & 35 \\ 60 & 47 \\ 67 & 54 \\ 72 & 57 \\ 75 & 63 \\ 77 & 67\end{array}$

$\begin{array}{rr}15 & 19.4 \\ 27 & 24.3 \\ 32 & 27.8 \\ 33 & 29.3 \\ 38 & 30.3 \\ 39 & 31.4 \\ \mathrm{C} 98 \mathrm{P} & \mathrm{C} 98 \mathrm{Q} \\ 51 & 33.3 \\ 50 & 33.7 \\ 52 & 33.8 \\ 53 & 34.5\end{array}$

Males

$1975 / 76$

$1985 / 86$

$1989 / 91$

$1992 / 94$

$1995 / 97^{2}$

GB90 C98

C98K

66 C98L

$98 \mathrm{~L}$
82
81
82

C98M

$\begin{array}{rr}\mathrm{C} 98 \mathrm{~N} & \mathrm{C} 98 \mathrm{O} \\ 82 & 74\end{array}$

$\begin{array}{ll}82 & 74 \\ 82 & 76 \\ 82 & 75\end{array}$

(millions

$1998 / 00$

2005

2006

2007

2008

$\begin{array}{ll}69 & 36 \\ 74 & 37 \\ 80 & 52 \\ 81 & 54 \\ 81 & 50 \\ 82 & 44\end{array}$

$\begin{array}{ll}36 & 78 \\ 37 & 73 \\ 52 & 82 \\ 54 & 83 \\ 50 & 80 \\ 44 & 80\end{array}$

$\begin{array}{ll}85 & 83 \\ 86 & 87 \\ 88 & 89 \\ 91 & 88 \\ 88 & 89 \\ 89 & 91\end{array}$

75
81
85
88
89
88

58
72
78
81
83
83

32
51
58
59
65
65

$698 \times$
73
76
75
75

13.4

$\begin{array}{rrrr}\text { GB9P } & \text { C98R } & \text { C98S } & \text { C98 } \\ 81 & 37 & 69 & 86 \\ 81 & 37 & 71 & 86 \\ 80 & 41 & 69 & 86 \\ 81 & 38 & 67 & 87\end{array}$

$\begin{array}{rr}98 T & \text { C98 } \\ 86 & 9 \\ 86 & 89 \\ 86 & 88 \\ 87 & 89\end{array}$

C98V

C98W

88
90
87
90

16.7

17.0

17.2

17.2
17.4

Females

$1975 / 76$

$1985 / 86$

$1989 / 91$

$1992 / 94$

$1995 / 97^{2}$

$1998 / 00$

$\begin{array}{ll}29 & 2 \\ 41 & 29 \\ 49 & 35 \\ 54 & 4 \\ 57 & 36 \\ 60 & 38\end{array}$

$\begin{array}{ll}20 & 43 \\ 29 & 54 \\ 35 & 64 \\ 42 & 68 \\ 36 & 67 \\ 38 & 69\end{array}$

$\begin{array}{ll}43 & 48 \\ 54 & 6 \\ 64 & 67 \\ 68 & 73 \\ 67 & 74 \\ 69 & 78\end{array}$

$\begin{array}{ll}48 & 37 \\ 62 & 56 \\ 67 & 66 \\ 73 & 70 \\ 74 & 73 \\ 78 & 76\end{array}$

$\begin{array}{ll}24 & 15 \\ 41 & 24 \\ 49 & 33 \\ 57 & 37 \\ 62 & 45 \\ 67 & 53\end{array}$

$\begin{array}{rr}4 & 6.0 \\ 11 & 9.2 \\ 15 & 11.1 \\ 16 & 12.2 \\ 21 & 13.1 \\ 22 & 14.0\end{array}$

2005

2006

2007

2008

GB9Q C98Z

$\begin{array}{lll}\mathrm{C} 992 & \mathrm{C} 993 & \mathrm{C994}\end{array}$

C995 $\quad$ C996

\begin{tabular}{rrrrr}
63 & C98Z & C992 & C993 & C994 \\
63 & 27 & 62 & 77 & 79 \\
63 & 31 & 63 & 78 & 79 \\
65 & 34 & 62 & 76 & 78 \\
\hline
\end{tabular}

$\begin{array}{rr}73 & 61 \\ 74 & 63 \\ 74 & 63 \\ 75 & 67\end{array}$

C997 $\quad$ C998

$\begin{array}{rrr} & \mathrm{C} 997 & \\ 61 & 35 & 15.2\end{array}$

$\begin{array}{lll}63 & 31 & 15.3 \\ 63 & 36 & 15.4 \\ 67 & 36 & 15.8\end{array}$

1 See chapter text.

2 Based on combined survey data sources - Family Expenditure Survey,

ONS; General Household Survey, ONS and National Travel Survey, DfT. 


\begin{tabular}{|c|c|c|c|c|c|}
\hline \multicolumn{6}{|c|}{ Percentages and millions } \\
\hline & No car & One car & $\begin{array}{l}\text { Two } \\
\text { cars }\end{array}$ & $\begin{array}{l}\text { Three or } \\
\text { more cars }\end{array}$ & $\begin{array}{r}\text { Total } \\
\text { (millions) }\end{array}$ \\
\hline & ZCGA & ZCGB & ZCGC & ZCGD & ZCGE \\
\hline 1997 & 30 & 45 & 21 & 5 & 23.1 \\
\hline 1998 & 28 & 44 & 23 & 5 & 23.3 \\
\hline 1999 & 28 & 44 & 22 & 5 & 23.5 \\
\hline 2000 & 27 & 45 & 23 & 5 & 23.7 \\
\hline 2001 & 26 & 45 & 23 & 5 & 23.9 \\
\hline 2002 & 26 & 44 & 24 & 5 & 24.2 \\
\hline 2003 & 26 & 44 & 25 & 5 & 24.4 \\
\hline 2004 & 25 & 44 & 25 & 5 & 24.6 \\
\hline 2005 & 25 & 44 & 25 & 5 & .. \\
\hline 2006 & 24 & 44 & 26 & 6 & 25.1 \\
\hline \multirow[t]{2}{*}{2007} & 24 & 44 & 26 & 6 & .. \\
\hline & & No car & One car & $\begin{array}{l}\text { Two or } \\
\text { more cars }\end{array}$ & Total \\
\hline
\end{tabular}

Government Office Regions, $2007^{2}$

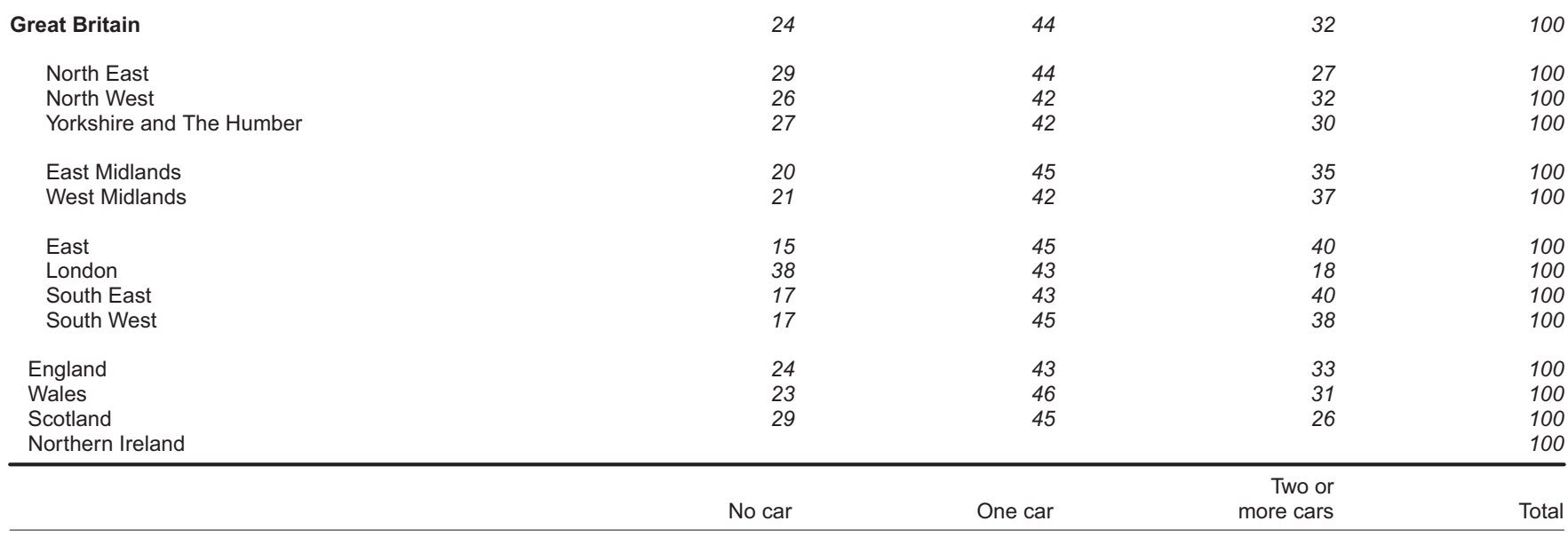

Area type, 2008

\begin{tabular}{|c|c|c|c|c|}
\hline Great Britain & 25 & 43 & 32 & 100 \\
\hline London & 43 & 40 & 17 & 100 \\
\hline Metropolitan areas & 32 & 41 & 26 & 100 \\
\hline Over 250,000 & 23 & 45 & 32 & 100 \\
\hline $25,000-250,000$ & 24 & 44 & 32 & 100 \\
\hline $10,000-25,000$ & 23 & 45 & 32 & 100 \\
\hline $3,000-10,000$ & 16 & 42 & 42 & 100 \\
\hline Rural areas & 10 & 43 & 47 & 100 \\
\hline
\end{tabular}


Numbers

\begin{tabular}{|c|c|c|c|c|c|c|c|c|c|c|c|c|}
\hline & & 1998 & 1999 & 2000 & $2001^{3}$ & 2002 & $2003^{4}$ & 2004 & 2005 & 2006 & 2007 & 2008 \\
\hline $\begin{array}{l}\text { Private light goods, etc } \\
\text { Motorcycles, Scooters and mopeds }\end{array}$ & $\begin{array}{ll}\mathrm{K} & \mathrm{KA} \\
\mathrm{K} & \mathrm{KB}\end{array}$ & $\begin{array}{r}584706 \\
11663\end{array}$ & $\begin{array}{r}608316 \\
13087\end{array}$ & $\begin{array}{r}615180 \\
14116\end{array}$ & $\begin{array}{r}644968 \\
15205\end{array}$ & $\begin{array}{r}666731 \\
17598\end{array}$ & $\begin{array}{r}711913 \\
23820\end{array}$ & $\begin{array}{r}737198 \\
24533\end{array}$ & $\begin{array}{r}765061 \\
25998\end{array}$ & $\begin{array}{r}800969 \\
27083\end{array}$ & $\begin{array}{r}840621 \\
28150\end{array}$ & $\begin{array}{r}857044 \\
28180\end{array}$ \\
\hline $\begin{array}{l}\text { Public road passenger vehicles }{ }^{2} \text { : } \\
\text { Taxis, buses,coaches } \\
\text { Buses, coaches ( } 9 \text { seats or more) }\end{array}$ & 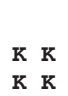 & 2175 & 2204 & 2266 & 2315 & 2322 & 2353 & 2378 & 2566 & 2670 & 2865 & 2951 \\
\hline Total & $\mathrm{K}$ KC & 2175 & 2204 & 2266 & 2315 & 2322 & 2353 & 2378 & 2566 & 2670 & 2865 & 2951 \\
\hline $\begin{array}{l}\text { General (HGV) goods vehicles: } \\
\text { Agricultural tractors and engines, etc }{ }^{3} \\
\text { Other }\end{array}$ & $\begin{array}{ll}\mathbf{K} & \mathbf{K} \\
\mathbf{K} & \mathbf{K M} \\
\mathbf{K} & \mathbf{K}\end{array}$ & $\begin{array}{r}18312 \\
5906 \\
1193\end{array}$ & $\begin{array}{r}17075 \\
5505 \\
1446\end{array}$ & $\begin{array}{r}17864 \\
5048 \\
1287\end{array}$ & $\begin{array}{r}19415 \\
4901 \\
1366\end{array}$ & $\begin{array}{r}20244 \\
5731 \\
1347\end{array}$ & $\begin{array}{r}22100 \\
7503 \\
1671\end{array}$ & $\begin{array}{r}23062 \\
8674 \\
1794\end{array}$ & $\begin{array}{r}23517 \\
9584 \\
1898\end{array}$ & $\begin{array}{r}24806 \\
10586 \\
2039\end{array}$ & $\begin{array}{r}25785 \\
12817 \\
2125\end{array}$ & $\begin{array}{r}25136 \\
14326 \\
2232\end{array}$ \\
\hline $\begin{array}{l}\text { Vehicles exempt from duty: } \\
\text { Government owned } \\
\text { Other: }\end{array}$ & $\mathrm{K} \quad \mathrm{KP}$ & 3785 & 4032 & 3822 & 6427 & 6383 & 6172 & 6116 & 6367 & 7315 & 9655 & 6902 \\
\hline $\begin{array}{l}\text { Ambulances } \\
\text { Fire engines } \\
\text { Other exempt }\end{array}$ & $\begin{array}{ll}\mathrm{K} & \mathrm{KQ} \\
\mathrm{K} & \mathrm{KR} \\
\mathrm{K} & \mathrm{KS}\end{array}$ & $\begin{array}{r}425 \\
285 \\
66981\end{array}$ & $\begin{array}{r}417 \\
286 \\
68277\end{array}$ & $\begin{array}{r}452 \\
290 \\
70405\end{array}$ & $\begin{array}{r}318 \\
181 \\
72209\end{array}$ & $\begin{array}{r}299 \\
174 \\
73648\end{array}$ & $\begin{array}{r}325 \\
170 \\
76715\end{array}$ & $\begin{array}{r}355 \\
178 \\
78973\end{array}$ & $\begin{array}{r}355 \\
179 \\
81874\end{array}$ & $\begin{array}{r}388 \\
166 \\
82655\end{array}$ & $\begin{array}{r}378 \\
155 \\
85738\end{array}$ & $\begin{array}{r}390 \\
142 \\
87093\end{array}$ \\
\hline Total & к ко & 71476 & 73012 & 74969 & 79135 & 80504 & 83382 & 85622 & 88775 & 90524 & 95926 & 94527 \\
\hline Total & $\mathrm{K}$ KT & 695431 & 720645 & 730730 & 767305 & 794477 & 852742 & 883261 & 917399 & 958677 & 1008289 & 1024396 \\
\hline
\end{tabular}

1 Licences current at 31 December.

2 Tax class change from 'Hackney' to 'Bus' with effect from July 2005. Only

Source: Driver and Vehicle Agency: 02870346903

Vehicles with 9 or more seats are included in 'Bus' class. Vehicles with 8

seats or less previously recorded in 'Hackney ' class moved into 'Private

Light Goods' class.

3 Taxation classes have been revised

4 New Tax Class 36 introduced.

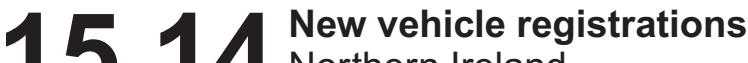 Northern Ireland}

\begin{tabular}{|c|c|c|c|c|c|c|c|c|c|c|c|c|c|}
\hline & & & 1998 & 1999 & 2000 & 2001 & 2002 & 2003 & 2004 & 2005 & 2006 & 2007 & 2008 \\
\hline Private cars & $\mathbf{K}$ & $\mathbf{A}$ & 91141 & 89078 & 84973 & 88592 & 83402 & 87506 & 85190 & 86366 & 91224 & 97346 & 7886 \\
\hline Motorcycles & $\mathbf{K}$ & B & 4307 & 5310 & 6010 & 5591 & 5596 & 6804 & 4601 & 4648 & 4289 & 4477 & 398 \\
\hline Public road passenger vehicles & $\mathbf{K}$ & C & 486 & 568 & 565 & 451 & 439 & 609 & 467 & 621 & 677 & 629 & \\
\hline \multicolumn{14}{|l|}{$\begin{array}{l}\text { Goods vehicles: } \\
\text { General haulage vehicles: }\end{array}$} \\
\hline 3.5 tonnes and over & $\mathbf{K}$ & & 3572 & 3697 & 3502 & 4534 & 3669 & 4059 & 3987 & 3768 & 4080 & 3676 & 292 \\
\hline Agricultural tractors ${ }^{1}$ & K & M & 971 & 987 & 1313 & 301 & 1 & 9 & 2 & 2 & 8 & - & \\
\hline Vehicles exempt from duty & $\mathbf{K}$ & $\mathbf{R}$ & 10718 & 11081 & 10789 & 12126 & 12515 & 11907 & 12881 & 13987 & 13031 & 14083 & 1484 \\
\hline General haulage and special types & & AG & .. & .. & .. & .. & 15 & 12 & 11 & 16 & 32 & 46 & \\
\hline Total & $\mathbf{K}$ & $\mathbf{s}$ & 121302 & 121777 & 119769 & 124869 & 117644 & 122398 & 118229 & 121708 & 126798 & 134112 & 11276 \\
\hline
\end{tabular}

1 Agricultural tractors driven on public roads. From April 2001 tractors were Source: Driver and Vehicle Agency : 02870346903 exempt.

\subsection{Local bus services: passenger journeys by area: 1998/99-2008/09}

\begin{tabular}{|c|c|c|c|c|c|c|c|c|c|c|c|c|}
\hline & & $\begin{array}{r}1998 \\
/ 99\end{array}$ & $\begin{array}{r}1999 \\
100\end{array}$ & $\begin{array}{r}2000 \\
101\end{array}$ & $\begin{array}{r}2001 \\
102\end{array}$ & $\begin{array}{r}2002 \\
103\end{array}$ & $\begin{array}{r}2003 \\
104\end{array}$ & $\begin{array}{r}2004 \\
105\end{array}$ & $\begin{array}{r}2005 \\
106\end{array}$ & $\begin{array}{c}2006^{1} \\
107\end{array}$ & $\begin{array}{c}2007^{2} \\
108\end{array}$ & $\begin{array}{r}2008^{2} \\
109\end{array}$ \\
\hline Great Britain & $\mathrm{zC} \mathrm{T}$ & 4350 & 4376 & 4420 & 4455 & 4550 & 4681 & 4737 & 4791 & 5097 & 5163 & 5233 \\
\hline London & $\mathrm{KI} \mathrm{S}$ & 1266 & 1294 & 1347 & 1422 & 1527 & 1692 & 1802 & 1881 & 1993 & 2089 & 2149 \\
\hline English Metropolitan Counties & KI T & 1256 & 1213 & 1203 & 1196 & 1182 & 1162 & 1128 & 1111 & 1141 & 1104 & 1111 \\
\hline English other areas & $\mathrm{KI} \mathrm{U}$ & 1286 & 1297 & 1292 & 1263 & 1255 & 1233 & 1210 & 1204 & 1336 & 1328 & 1335 \\
\hline All outside London & $\mathrm{zC} \mathbf{s}$ & 3084 & 3082 & 3073 & 3033 & 3023 & 2989 & 2935 & 2910 & 3104 & 3074 & 3084 \\
\hline England & $\mathbf{z C} \mathbf{R}$ & 3808 & 3804 & 3842 & 3881 & 3964 & 4087 & 4140 & 4196 & 4470 & 4522 & 4594 \\
\hline Scotland & $\mathrm{KI} \mathrm{V}$ & 424 & 455 & 458 & 466 & 471 & 478 & 479 & 477 & 506 & 517 & 515 \\
\hline Wales & $\mathrm{KI} \mathrm{W}$ & 118 & 117 & 119 & 108 & 115 & 116 & 118 & 118 & 122 & 124 & 124 \\
\hline
\end{tabular}

1 There is a break in the series after 2006/07.

Source: Department for Transport 02079443076 
Indices $(1995=100)$

\begin{tabular}{|c|c|c|c|c|c|c|c|c|c|c|c|c|}
\hline & & $\begin{array}{r}1998 \\
199 \\
\end{array}$ & $\begin{array}{r}1999 \\
100 \\
\end{array}$ & $\begin{array}{r}2000 \\
101 \\
\end{array}$ & $\begin{array}{r}2001 \\
102 \\
\end{array}$ & $\begin{array}{r}2002 \\
103 \\
\end{array}$ & $\begin{array}{r}2003 \\
104 \\
\end{array}$ & $\begin{array}{r}2004 \\
105 \\
\end{array}$ & $\begin{array}{r}2005 \\
106 \\
\end{array}$ & $\begin{array}{r}2006 \\
107 \\
\end{array}$ & $\begin{array}{r}2007 \\
108 \\
\end{array}$ & $\begin{array}{r}2008 \\
109 \\
\end{array}$ \\
\hline Great Britain & KNEU & 117.1 & 121.8 & 126.4 & 130.6 & 134.5 & 139.2 & 146.5 & 157.5 & 159.0 & 167.6 & 176.2 \\
\hline London & KNEP & 113.8 & 117.2 & 117.3 & 115.5 & 114.8 & 116.9 & 126.8 & 139.7 & 151.5 & 159.5 & 160 \\
\hline English Metropolitan Counties & KILD & 117.9 & 123.5 & 128.6 & 135.5 & 140.7 & 146.7 & 153.3 & 166.0 & 168.3 & 178.3 & 190 \\
\hline English other areas & KILE & 117.3 & 122.6 & 129.2 & 136.1 & 142.4 & 149.0 & 155.9 & 166.2 & 159.5 & 168.0 & 178 \\
\hline All outside London & $\mathrm{ZCEQ}$ & 118.2 & 123.2 & 129.0 & 135.1 & 140.4 & 146.0 & 152.3 & 162.2 & 160.7 & 169.1 & 180 \\
\hline England & ZCEP & 116.4 & 121.4 & 125.8 & 130.3 & 134.3 & 139.4 & 147.2 & 159.4 & 160.1 & 169.1 & 177 \\
\hline Scotland & KILF & 121.2 & 124.1 & 129.1 & 131.1 & 133.8 & 136.1 & 140.0 & 143.9 & 151.0 & 155.7 & 166 \\
\hline Wales & KILG & 116.0 & 121.9 & 128.4 & 135.7 & 142.3 & 147.2 & 153.7 & 159.9 & 169.7 & 177.8 & 188 \\
\hline $\begin{array}{l}\text { Retail Prices Index } \\
(1995=100)\end{array}$ & KNEV & 109.9 & 111.6 & 114.9 & 116.6 & 119.1 & 122.4 & 126.2 & 129.5 & 134.4 & 139.9 & 144 \\
\hline
\end{tabular}

\section{$15.7 \begin{aligned} & \text { Road accident casualties: by road user type and severity } \\ & \text { Great Britain }\end{aligned}$}

\begin{tabular}{|c|c|c|c|c|c|c|c|c|c|c|c|c|}
\hline & & & & & & & & & & & & Numbers \\
\hline & & 1998 & 1999 & 2000 & 2001 & 2002 & 2003 & 2004 & 2005 & 2006 & 2007 & 2008 \\
\hline hild pedestrians ${ }^{1}$ : & & & & & & & & & & & & \\
\hline Killed & $\mathrm{ZCDH}$ & 103 & 107 & 107 & 107 & 79 & 74 & 77 & 63 & 71 & 57 & \\
\hline Killed or seriously injured & KIJS & 3737 & 3457 & 3226 & 3144 & 2828 & 2381 & 2339 & 2134 & 2025 & 1899 & $17 \varepsilon$ \\
\hline All severities & ZCDI & 17971 & 16876 & 16184 & 15819 & 14231 & 12544 & 12234 & 11250 & 10131 & 9527 & 864 \\
\hline Adult pedestrians ${ }^{2}$ : & & & & & & & & & & & & \\
\hline Killed & $\mathrm{ZCDJ}$ & 803 & 760 & 750 & 712 & 688 & 695 & 589 & 604 & 602 & 585 & 51 \\
\hline Killed or seriously injured & KIJT & 6592 & 6221 & 6112 & 5745 & 5644 & 5422 & 5005 & 4847 & 4894 & 4900 & 472 \\
\hline All severities & $\mathrm{ZCDK}$ & 25827 & 24806 & 24481 & 23463 & 23258 & 22531 & 21404 & 20725 & 19774 & 19676 & 1901 \\
\hline Child pedal cyclists $^{1}$ : & & & & & & & & & & & & \\
\hline Killed & $\mathrm{ZCDL}$ & 32 & 36 & 27 & 25 & 22 & 18 & 25 & 20 & 31 & 13 & \\
\hline Killed or seriously injured & KIJU & 915 & 950 & 758 & 674 & 594 & 595 & 577 & 527 & 503 & 522 & 417 \\
\hline All severities & $\mathrm{ZCDM}$ & 6930 & 7290 & 6260 & 5451 & 4809 & 4769 & 4682 & 4286 & 3765 & 3633 & 330 \\
\hline Adult pedal cyclists ${ }^{2}$ : & & & & & & & & & & & & \\
\hline Killed & $\mathrm{ZCDN}$ & 126 & 135 & 98 & 111 & 107 & 95 & 109 & 127 & 115 & 122 & 103 \\
\hline Killed or seriously injured & $\mathrm{KIJV}$ & 2345 & 2172 & 1954 & 1951 & 1801 & 1776 & 1697 & 1787 & 1898 & 1994 & 210 \\
\hline All severities & $\mathrm{ZCDO}$ & 15326 & 14834 & 13630 & 12974 & 11712 & 11643 & 11366 & 11637 & 11911 & 12050 & 125 \\
\hline Motorcyclists ${ }^{3}$ and passe & & & & & & & & & & & & \\
\hline Killed & $\mathrm{ZCDP}$ & 498 & 547 & 605 & 583 & 609 & 693 & 585 & 569 & 599 & 588 & $4 \varsigma$ \\
\hline Killed or seriously injured & $\mathrm{ZCDQ}$ & 6442 & 6908 & 7374 & 7305 & 7500 & 7652 & 6648 & 6508 & 6484 & 6737 & 60 \\
\hline All severities & BMDH & 24610 & 26192 & 28212 & 28810 & 28353 & 28411 & 25641 & 24824 & 23326 & 23459 & 215 \\
\hline Car drivers and passeng & & & & & & & & & & & & \\
\hline Killed & $\mathrm{zCDS}$ & 1696 & 1687 & 1665 & 1749 & 1747 & 1769 & 1671 & 1675 & 1612 & 1432 & 1257 \\
\hline Killed or seriously injured & $\mathrm{ZCDT}$ & 21676 & 20368 & 19719 & 19424 & 18728 & 17291 & 16144 & 14617 & 14254 & 12967 & 11968 \\
\hline All severities & $\mathrm{ZCDU}$ & 210474 & 205735 & 206799 & 202802 & 197425 & 188342 & 183858 & 178302 & 171000 & 161433 & 14918 \\
\hline Bus/coach drivers and $p$ & & & & & & & & & & & & \\
\hline Killed & $\mathrm{zCDV}$ & 18 & 11 & 15 & 14 & 19 & 11 & 20 & 9 & 19 & 12 & \\
\hline Killed or seriously injured & KCUZ & 631 & 611 & 578 & 562 & 551 & 500 & 488 & 363 & 426 & 455 & 4 \\
\hline All severities & ZCDW & 9839 & 10252 & 10088 & 9884 & 9005 & 9068 & 8820 & 7920 & 7253 & 7079 & 69 \\
\hline LGV driver & & & & & & & & & & & & \\
\hline Killed & $\mathrm{zCDx}$ & 67 & 65 & 66 & 64 & 70 & 72 & 62 & 54 & 52 & 58 & \\
\hline Killed or seriously injured & $\mathrm{ZCDY}$ & 949 & 867 & 813 & 811 & 780 & 765 & 631 & 587 & 564 & 494 & 4 \\
\hline All severities & $\mathrm{zCDZ}$ & 7672 & 7124 & 7007 & 7304 & 7007 & 6897 & 6166 & 6048 & 5914 & 5340 & 49 \\
\hline HGV drivers and passe & & & & & & & & & & & & \\
\hline Killed & $\mathrm{ZCEA}$ & 60 & 52 & 55 & 54 & 63 & 44 & 47 & 55 & 39 & 52 & \\
\hline Killed or seriously injured & ZCEB & 560 & 540 & 571 & 500 & 524 & 429 & 406 & 395 & 383 & 363 & \\
\hline All severities & $\mathrm{ZCEC}$ & 3444 & 3484 & 3597 & 3388 & 3178 & 3061 & 2883 & 2843 & 2530 & 2476 & 19 \\
\hline All road & & & & & & & & & & & & \\
\hline Killed & BMDC & 3421 & 3423 & 3409 & 3450 & 3431 & 3508 & 3221 & 3201 & 3172 & 2946 & 25 \\
\hline Killed or seriously injured & $\mathrm{ZCEE}$ & 44255 & 42545 & 41564 & 40560 & 39407 & 37215 & 34351 & 32155 & 31845 & 30720 & 285 \\
\hline All severities & BMDA & 325212 & 320310 & 320283 & 313309 & 302605 & 290607 & 280840 & 271017 & 258404 & 247780 & 2309 \\
\hline
\end{tabular}

1 Casualities aged $0-15$.

2 Casualties aged 16 and over.

3 Includes mopeds and scooters.

4 Includes other motor or non-motor vehicle users, and unknown road user type and casualty age. 
Transport and communications 15.18 Freight transport by road: goods moved by goods vehicles over 3.5 tonnes ${ }^{1}$

Billion tonne kilometres

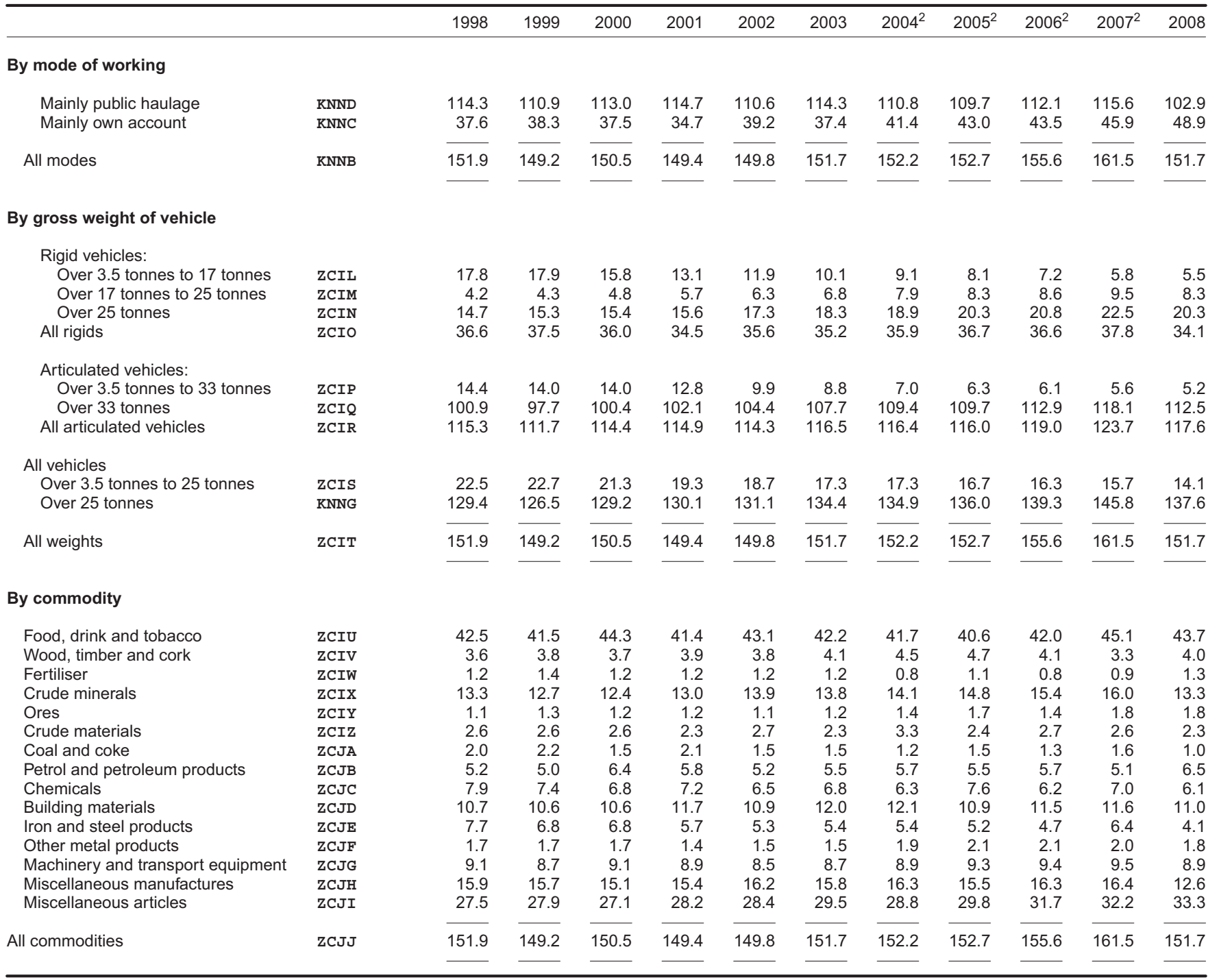

1 Rigid vehicles or articulated vehicles (tractive unit and trailer) with gross ve-

Source: Department for Transport 02079443180 hicle weight over 3.5 tonnes.

2 Figures for 2004 onwards are not fully comparable with those for 2003 and earlier years. Detailed comparisons should therefore be made with caution. 
Million tonnes

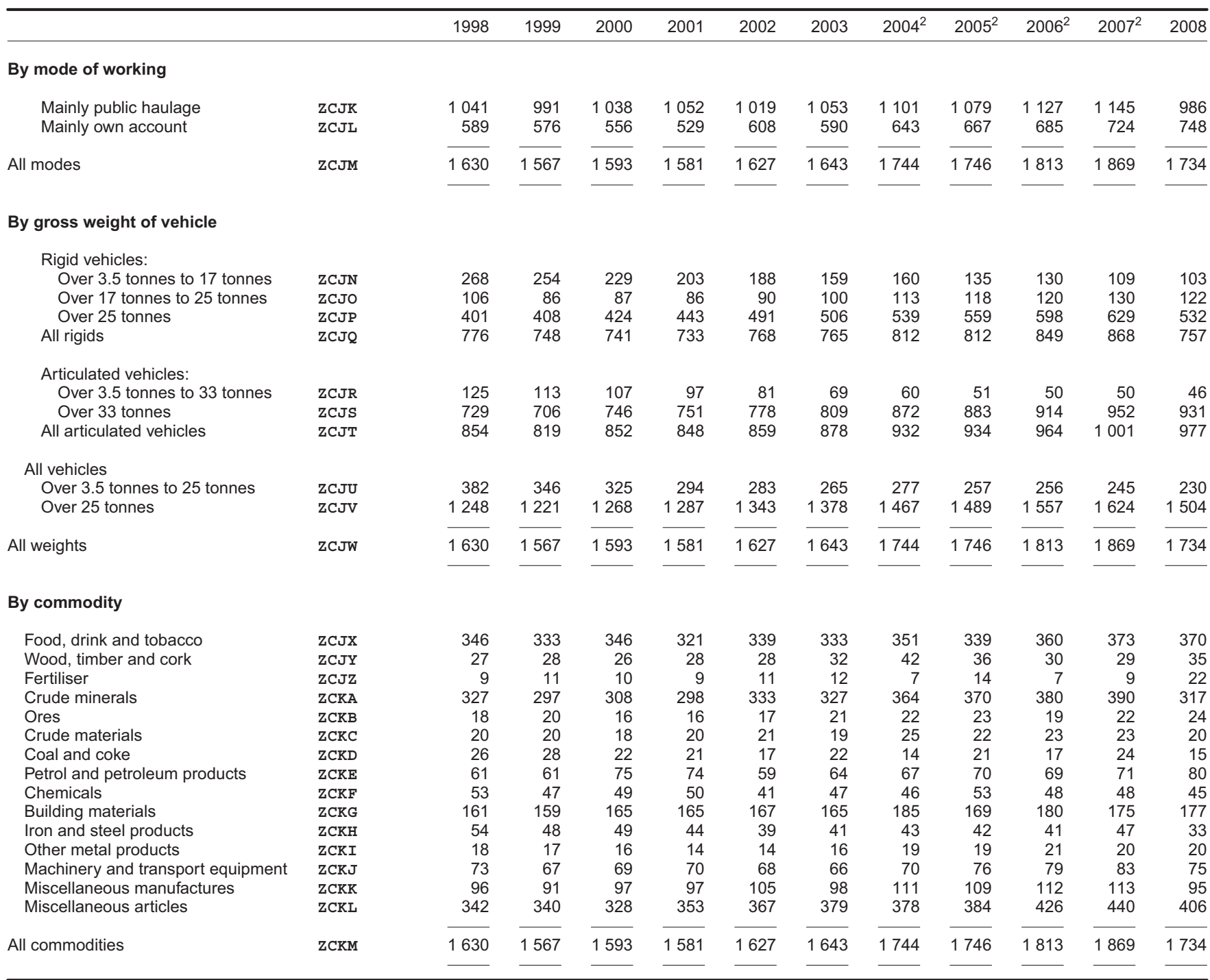

1 Rigid vehicles or articulated vehicles (tractive unit and trailer) with gross veSource: Department for Transport 02079443180 hicle weight over 3.5 tonnes.

2 Figures for 2004 onwards are not fully comparable with those for 2003 and earlier years. Detailed comparison should therefore be made with caution. 


\begin{tabular}{|c|c|c|c|c|c|c|c|c|c|c|c|c|}
\hline & & $\begin{array}{r}1998 \\
/ 99\end{array}$ & $\begin{array}{r}1999 \\
100\end{array}$ & $\begin{array}{r}2000 \\
/ 01\end{array}$ & $\begin{array}{r}2001 \\
/ 02\end{array}$ & $\begin{array}{r}2002 \\
103\end{array}$ & $\begin{array}{r}2003 \\
104\end{array}$ & $\begin{array}{r}2004 \\
105\end{array}$ & $\begin{array}{r}2005 \\
106\end{array}$ & $\begin{array}{r}2006 \\
107\end{array}$ & $\begin{array}{r}2007 \\
108\end{array}$ & $\begin{array}{r}2008 \\
109\end{array}$ \\
\hline \multicolumn{13}{|l|}{ Passenger journeys (millions) } \\
\hline National Rail network ${ }^{1}$ & $\mathrm{ZCKN}$ & 892 & 931 & 957 & 960 & 976 & 1012 & 1045 & 1082 & 1151 & 1232 & 1274 \\
\hline London Underground & KNOE & 866 & 927 & 970 & 953 & 942 & 948 & 976 & 970 & 1040 & 1096 & 1089 \\
\hline Docklands Light Railway & ZCKO & 28 & 31 & 38 & 41 & 46 & 48 & 50 & 54 & 64 & 67 & 68 \\
\hline Glasgow Underground & $\mathrm{ZCKP}$ & 15 & 15 & 14 & 14 & 13 & 13 & 13 & 13 & 13 & 14 & 14 \\
\hline Tyne and Wear Metro ${ }^{2}$ & $\mathrm{ZCKQ}$ & 34 & 33 & 33 & 33 & 37 & 38 & 37 & 36 & 38 & 40 & 41 \\
\hline Blackpool trams ${ }^{3}$ & EL9L & 4 & 4 & 4 & 5 & 4 & 4 & 4 & 4 & 3 & 3 & 2 \\
\hline Midland Metro ${ }^{5}$ & ZCKR & - & 5 & 5 & 5 & 5 & 5 & 5 & 5 & 5 & 5 & 5 \\
\hline Croydon Tramlink ${ }^{6}$ & GEOE & - & - & 15 & 18 & 19 & 20 & 22 & 23 & 25 & 27 & 27 \\
\hline Sheffield Supertram & ZCKT & 10 & 11 & 11 & 11 & 12 & 12 & 13 & 13 & 14 & 15 & 15 \\
\hline Nottingham $\mathrm{NET}^{7}$ & C3MI & - & - & - & - & - & - & 8 & 10 & 10 & 10 & 10 \\
\hline All rail & $\mathrm{ZCKU}$ & 1862 & 1971 & 2065 & 2059 & 2072 & 2119 & 2193 & 2229 & 2383 & 2529 & 2566 \\
\hline All light rail & GENZ & 104 & 113 & 138 & 146 & 154 & 160 & 172 & 177 & 192 & 201 & 203 \\
\hline \multicolumn{13}{|c|}{ Passenger revenue ( $£$ million at current prices) } \\
\hline Docklands Light Railway & $\mathrm{zCKV}$ & 20 & 22 & 29 & 32 & 36 & 37 & 40 & 46 & 54 & 62 & 63 \\
\hline Glasgow Underground & ZCKW & 9 & 10 & 10 & 10 & 10 & 10 & 11 & 11 & 13 & 13 & 14 \\
\hline Tyne and Wear Metro & $\mathrm{zCKX}$ & 23 & 24 & 24 & 25 & 29 & 31 & 33 & 34 & 38 & 32 & 32 \\
\hline Blackpool trams & EL9M & 4 & 4 & 4 & 5 & 5 & 4 & 4 & 4 & 5 & 4 & 3 \\
\hline Manchester Metrolink & ZCKZ & .. & .. & 18 & 20 & 20 & 21 & 22 & 23 & 24 & 22 & 23 \\
\hline Midland Metro & ZCKY & - & .. & 3 & 4 & 5 & 5 & 5 & 6 & 6 & 4 & 5 \\
\hline Croydon Tramlink & GEOF & - & - & 12 & 13 & 15 & 16 & 18 & 19 & 20 & 15 & 14 \\
\hline Sheffield Supertram & ZCLA & 6 & 7 & 7 & 8 & 10 & 9 & 11 & 10 & 13 & 11 & 12 \\
\hline Nottingham NET & $\mathrm{C} 3 \mathrm{MJ}$ & - & - & - & - & - &.. & 6 & 7 & 8 & 7 & 8 \\
\hline All rail & ZCLB & 3789 & 4493 & 4650 & 4815 & 4931 & 5197 & 5550 & 5963 & 6609 & 7251 & 7792 \\
\hline All light rail & GEOA & 69 & 63 & 108 & 117 & 130 & 135 & 151 & 161 & 180 & 171 & 173 \\
\hline \multicolumn{13}{|l|}{ Passenger kilometres (millions) } \\
\hline National Rail network & KNDZ & 36280 & 38472 & 38179 & 39141 & 39678 & 40906 & 41762 & 43211 & 46218 & 49007 & 50698 \\
\hline London Underground & KNOI & 6716 & 7171 & 7470 & 7451 & 7367 & 7340 & 7606 & 7586 & 7947 & 8352 & 8646 \\
\hline Docklands Light Railway & ZCLC & 144 & 172 & 200 & 207 & 232 & 235 & 245 & 257 & 301 & 326 & 318 \\
\hline Glasgow Underground & ZCLD & 47 & 47 & 46 & 44 & 43 & 43 & 43 & 42 & 42 & 46 & 45 \\
\hline Tyne and Wear Metro & ZCLE & 238 & 230 & 229 & 238 & 275 & 284 & 283 & 279 & 295 & 313 & 319 \\
\hline Croydon Tramlink & GEOG & - & - & 96 & 99 & 100 & 105 & 112 & 117 & 128 & 141 & 144 \\
\hline Sheffield Supertram & $\mathrm{ZCLH}$ & 35 & 37 & 38 & 39 & 40 & 42 & 44 & 44 & 42 & 44 & 45 \\
\hline Nottingham NET & C3MK & - & - & - & - & - & 2 & 37 & 42 & 43 & 44 & 42 \\
\hline All rail & ZCLI & 43577 & 46318 & 46479 & 47446 & 47965 & 49191 & 50401 & 51849 & 55285 & 58544 & 60535 \\
\hline All light rail & GEOB & 581 & 675 & 830 & 854 & 920 & 945 & 1033 & 1052 & 1120 & 1185 & 1191 \\
\hline \multicolumn{13}{|c|}{ Route kilometres open for passenger traffic (numbers) } \\
\hline National Rail network ${ }^{8}$ & $\mathrm{ZCLJ}$ & 15038 & 15038 & 15042 & 15042 & 15042 & 14883 & 14328 & 14356 & 14353 & 14484 & 14494 \\
\hline London Underground & ZCLK & 392 & 408 & 408 & 408 & 408 & 408 & 408 & 408 & 408 & 408 & 408 \\
\hline Docklands Light Railway & ZCLM & 22 & 26 & 26 & 26 & 26 & 26 & 26 & 30 & 31 & 32 & 33 \\
\hline Glasgow Underground & ZCLN & 11 & 11 & 11 & 11 & 11 & 11 & 11 & 10 & 10 & 10 & 10 \\
\hline Tyne and Wear Metro & ZCLO & 59 & 59 & 59 & 78 & 78 & 78 & 78 & 78 & 78 & 78 & 78 \\
\hline Blackpool trams & EL9O & 18 & 18 & 18 & 18 & 18 & 18 & 18 & 18 & 18 & 18 & 18 \\
\hline Manchester Metrolink & $\mathrm{ZCLQ}$ & 31 & 39 & 39 & 39 & 39 & 39 & 39 & 39 & 39 & 42 & 39 \\
\hline Midland Metro & ZCLP & - & 20 & 20 & 20 & 20 & 20 & 20 & 20 & 20 & 20 & 20 \\
\hline Croydon Tramlink & $\mathrm{GEOH}$ & - & - & 28 & 28 & 28 & 28 & 28 & 28 & 28 & 28 & 28 \\
\hline Sheffield Supertram & ZCLR & 29 & 29 & 29 & 29 & 29 & 29 & 29 & 29 & 29 & 29 & 29 \\
\hline Nottingham NET & C3ML & - & - & - & - & - & 14 & 14 & 15 & 14 & 14 & 14 \\
\hline All rail & ZCLS & 15600 & 15648 & 15680 & 15699 & 15699 & 15554 & 14999 & 15032 & 15028 & 15186 & 15171 \\
\hline All light rail & GEOC & 170 & 202 & 230 & 249 & 249 & 263 & 263 & 268 & 267 & 294 & 269 \\
\hline \multicolumn{13}{|l|}{ Stations served (numbers) } \\
\hline National Rail network & ZCLT & 2499 & 2503 & 2508 & 2508 & 2508 & 2507 & 2508 & 2510 & 2520 & 2516 & 2516 \\
\hline London Underground & KNOO & 269 & 274 & 274 & 274 & 274 & 274 & 274 & 274 & 273 & 268 & 270 \\
\hline Docklands Light Railway & ZCLU & 29 & 34 & 34 & 34 & 34 & 34 & 34 & 38 & 34 & 39 & 40 \\
\hline Glasgow Underground & $\mathrm{ZCLV}$ & 15 & 15 & 15 & 15 & 15 & 15 & 15 & 15 & 15 & 15 & 15 \\
\hline Tyne and Wear Metro & ZCLW & 46 & 46 & 46 & 58 & 58 & 58 & 58 & 59 & 59 & 60 & 60 \\
\hline Blackpool trams & EL9P & 124 & 124 & 124 & 124 & 124 & 124 & 124 & 124 & 121 & 121 & 121 \\
\hline Manchester Metrolink & ZCLY & 26 & 36 & 36 & 36 & 37 & 37 & 37 & 37 & 37 & 37 & 37 \\
\hline Midland Metro & $\mathrm{ZCLX}$ & - & 23 & 23 & 23 & 23 & 23 & 23 & 23 & 23 & 23 & 23 \\
\hline Croydon Tramlink & GEOI & - & - & 38 & 38 & 38 & 38 & 38 & 39 & 39 & 38 & 39 \\
\hline Sheffield Supertram & ZCLZ & 47 & 47 & 47 & 48 & 48 & 48 & 48 & 48 & 48 & 48 & 48 \\
\hline Nottingham NET & C3MM & - & - & - & - & - & 23 & 23 & 23 & 23 & 23 & 23 \\
\hline All rail & ZCLL & 3055 & 3102 & 3146 & 3145 & 3159 & 3181 & 3182 & 3190 & 3192 & 3188 & 3192 \\
\hline All light rail & GSOC & 287 & 325 & 363 & 376 & 377 & 400 & 400 & 406 & 399 & 404 & 406 \\
\hline
\end{tabular}


Billion tonne kilometres

\begin{tabular}{|c|c|c|c|c|c|c|c|c|c|c|c|c|}
\hline & & & $\begin{array}{c}1998^{1} \\
/ 99\end{array}$ & $\begin{array}{r}1999 \\
/ 00\end{array}$ & $\begin{array}{r}2000 \\
/ 01\end{array}$ & $\begin{array}{r}2001 \\
/ 02\end{array}$ & $\begin{array}{r}2002 \\
/ 03\end{array}$ & $\begin{array}{r}2003 \\
104\end{array}$ & $\begin{array}{r}2005 \\
106\end{array}$ & $\begin{array}{r}2006 \\
107\end{array}$ & $\begin{array}{r}2007 \\
108\end{array}$ & $\begin{array}{r}2008 \\
109\end{array}$ \\
\hline \multicolumn{13}{|c|}{ Freight moved by commodity } \\
\hline Coal & ZCGG & & 4.5 & 4.8 & 4.8 & 6.2 & 5.7 & 5.8 & 8.3 & 8.6 & 7.7 & 7.9 \\
\hline Metals & $\mathrm{ZCGH}$ & & 2.1 & 2.2 & 2.1 & 2.4 & 2.7 & 2.6 & 2.2 & 2.0 & 1.8 & 1.5 \\
\hline Construction & ZCGI & & 2.1 & 2.0 & 2.4 & 2.8 & 2.5 & 2.7 & 2.9 & 2.7 & 2.8 & 2.7 \\
\hline Oil and petroleum & $\mathrm{ZCGJ}$ & & 1.6 & 1.5 & 1.4 & 1.2 & 1.2 & 1.2 & 1.2 & 1.5 & 1.6 & 1.5 \\
\hline Other traffic & ZCGK & & 7.1 & 7.6 & 7.4 & 6.7 & 6.6 & 6.8 & 7.1 & 7.1 & 7.2 & 7.0 \\
\hline \multirow[t]{3}{*}{ All traffic } & VOxD & & 17.3 & 18.2 & 18.1 & 19.4 & 18.5 & 18.9 & 21.7 & 21.9 & 21.2 & 20.6 \\
\hline & & & & & & & & & & & Million & tonnes \\
\hline & & $\begin{array}{r}1998 \\
/ 99\end{array}$ & $\begin{array}{c}1999^{2} \\
100\end{array}$ & $\begin{array}{r}2000 \\
/ 01\end{array}$ & $\begin{array}{r}2001 \\
/ 02\end{array}$ & $\begin{array}{r}2002 \\
103\end{array}$ & $\begin{array}{r}2003 \\
104\end{array}$ & $\begin{array}{c}2004^{3} \\
105\end{array}$ & $\begin{array}{c}2005^{4} \\
106\end{array}$ & $\begin{array}{r}2006 \\
107\end{array}$ & $\begin{array}{c}2007^{5} \\
108\end{array}$ & $\begin{array}{r}2008 \\
109\end{array}$ \\
\hline Coal & ZCGL & 45.3 & 35.9 & 35.3 & 39.5 & 34.0 & 35.2 & 43.3 & 47.6 & 48.7 & 43.3 & 46.6 \\
\hline Metals & ZCGM & .. & .. & .. & .. & .. & .. & .. &.. & .. &.. & .. \\
\hline Construction & ZCGN & .. &.. & .. & .. & .. & .. & .. &.. & .. &.. & .. \\
\hline Oil and petroleum & $\mathrm{ZCGO}$ & & .. & .. & .. & .. & .. & .. & .. & .. & .. & .. \\
\hline Other traffic & ZCGP & 56.8 & 60.6 & 60.3 & 54.0 & 53.0 & 53.7 & 56.8 & 57.7 & $59 . \ddot{5}$ & 59.1 & 56.1 \\
\hline All traffic & voxe & 102.100 & 96.500 & 95.600 & 93.900 & 87.000 & 88.900 & 100.100 & 105.300 & 108.211 & 102.403 & 102.700 \\
\hline
\end{tabular}

1 There is a break in the series between 1998-99 and 1999-00 due to a Source: Rail :ORR : 02079448874 change in the source data.

2 Break in series from 1999/2000.

3 Break in series with most of the increase due to changes in the data collec-

tion method.

4 Break in the series from 2005/06 as some GB Railfreight tonnes lifted now

included.

5 Break in series from 2007/08 as GB Railfreight coal data now included.

Railways: permanent way and rolling stock

Northern Ireland

At end of year

Numbers

\begin{tabular}{|c|c|c|c|c|c|c|c|c|c|c|c|c|}
\hline & & 1998 & 1999 & 2000 & 2001 & 2002 & 2003 & 2004 & 2005 & 2006 & 2007 & 2008 \\
\hline Length of road open for traffic ${ }^{1}(\mathrm{Km})$ & KNRA & 335 & 335 & 356 & 334 & 334 & 334 & 299 & 299 & 299 & 299 & 299 \\
\hline \multicolumn{13}{|l|}{ Length of track open for traffic $(\mathrm{Km})$} \\
\hline Total & KNRB & 526 & 526 & 547 & 480 & 480 & 480 & 445 & 445 & 445 & 445 & 445 \\
\hline Running lines & KNRC & 484 & 484 & 505 & 464 & 464 & 464 & 427 & 427 & 427 & 427 & 427 \\
\hline Sidings (as single track) & KNRD & 42 & 42 & 42 & 16 & 16 & 16 & 18 & 18 & 18 & 18 & 18 \\
\hline \multicolumn{13}{|l|}{ Locomotives } \\
\hline \multicolumn{13}{|l|}{ Passenger carrying vehicles } \\
\hline Total & KNRF & 120 & 105 & 105 & 106 & 100 & 100 & 102 & 124 & 125 & 128 & 130 \\
\hline Rail motor vehicles: & & & & & & & & & & & & \\
\hline Diesel-electric, etc & KNRG & 28 & 30 & 30 & 29 & 28 & 28 & 28 & 70 & 85 & 84 & 84 \\
\hline \multicolumn{13}{|l|}{ Trailer carriages: } \\
\hline Total locomotive hauled & KNRH & 38 & 21 & 21 & 25 & 22 & 22 & 22 & 22 & 22 & 22 & 22 \\
\hline Ordinary coaches & KNRI & 36 & 19 & 19 & 23 & 20 & 20 & 20 & 20 & 20 & 20 & 20 \\
\hline Restaurant cars & KNRJ & 2 & 2 & 2 & 2 & 2 & 2 & 2 & 2 & 2 & 2 & 2 \\
\hline \multicolumn{13}{|l|}{ Rolling stock for maintenance } \\
\hline and repair & KNRT & 26 & 18 & 18 & 18 & 18 & 39 & 46 & 48 & 48 & 48 & 48 \\
\hline
\end{tabular}




\begin{tabular}{|c|c|c|c|c|c|c|c|c|c|c|c|c|c|}
\hline & & & 1998 & 1999 & 2000 & 2001 & 2002 & 2003 & 2004 & 2005 & 2006 & 2007 & 2008 \\
\hline \multirow{2}{*}{\multicolumn{14}{|c|}{ Maintenance of way and work }} \\
\hline & & & & & & & & & & & & & \\
\hline \multicolumn{14}{|l|}{ Material used: } \\
\hline Ballast & KNSA & $\begin{array}{c}\text { Thousand } \mathrm{m}^{2} \\
\text { Thousand }\end{array}$ & 38.5 & 40.0 & 47.0 & 80.0 & 40.0 & 130.0 & 70.0 & 90.0 & 30.0 & 15.0 & 10.0 \\
\hline Rails & KNSB & tonnes & 2.5 & 3.0 & 3.5 & 2.5 & 1.0 & 4.5 & 1.0 & 3.2 & 1.0 & 1.0 & \\
\hline Sleepers & KNSC & Thousands & 32.0 & 30.0 & 40.0 & 50.0 & 5.0 & 40.0 & 28.0 & 45.0 & 2.0 & 5.0 & \\
\hline Track renewed & KNSD & $\mathrm{Km}$ & 22.5 & 7.0 & 29.0 & 15.0 & 5.0 & 25.8 & 2.0 & 29.0 & 1.0 & - & \\
\hline \multicolumn{14}{|c|}{ Engine kilometres } \\
\hline Total $^{1}$ & KNSE & Thousand $\mathrm{Km}$ & 4100 & 4100 & 4100 & 4056 & 4056 & 4170 & 4110 & 3610 & 3900 & 3900 & 3900 \\
\hline \multicolumn{14}{|l|}{ Train kilometres: } \\
\hline Total & KNSF & " & 3670 & 3670 & 3670 & 3626 & 3626 & 3704 & 3610 & 3610 & 3900 & 3900 & 3900 \\
\hline Coaching & KNSG & " & 3666 & 3666 & 3666 & 3622 & 3622 & 3700 & 3610 & 3610 & 3900 & 3900 & 3900 \\
\hline Freight & KNSH & " & 4 & 4 & 4 & 4 & 4 & 4 & - & - & - & - & \\
\hline
\end{tabular}

1 Including shunting, assisting, light, departmental, maintenance and repair.

Sources: Department for Regional Development Northern Ireland: 02890540981

\subsection{4 $4^{\text {Min output of United Kingsom artilins }}$}

\begin{tabular}{|c|c|c|c|c|c|c|c|c|c|c|c|c|}
\hline & & & & & & & & & \multicolumn{4}{|c|}{ Available tonne kilometres (millions) } \\
\hline & & 1999 & 2000 & 2001 & 2002 & 2003 & 2004 & 2005 & 2006 & 2007 & 2008 & 2009 \\
\hline All services & KNTA & 42002 & 43379 & 42370 & 40550 & 42784 & 43883 & 48186 & 50391 & 54181 & 53348 & 49150 \\
\hline Percentage growth on previous year & KNTB & 5.0 & 3.6 & -2.4 & -4.3 & 5.5 & 2.6 & 9.8 & 4.4 & 7.5 & -1.6 & -7.9 \\
\hline Scheduled services & KNTC & 31815 & 32938 & 31866 & 30433 & 31513 & 32422 & 36937 & 38590 & 40971 & 41241 & 39207 \\
\hline Percentage growth on previous year & KNTD & 6.9 & 3.5 & -3.3 & -4.5 & 3.6 & 2.9 & 13.9 & 4.5 & 6.2 & 0.7 & -5.1 \\
\hline Non-scheduled services & KNTE & 10186 & 10440 & 10505 & 10117 & 11271 & 11461 & 11249 & 11801 & 13209 & 12077 & 9944 \\
\hline Percentage growth on previous year & KNTF & -0.7 & 4.1 & 0.6 & -3.7 & 11.4 & 1.7 & -1.8 & 4.3 & 11.9 & -8.6 & -17.7 \\
\hline
\end{tabular}

Source: Civil Aviation Authority: 02074536246

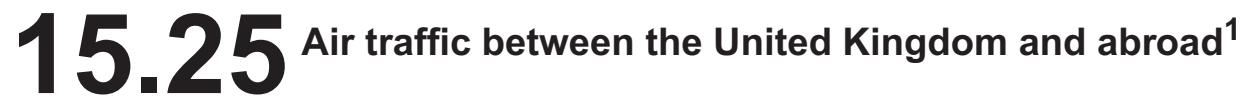

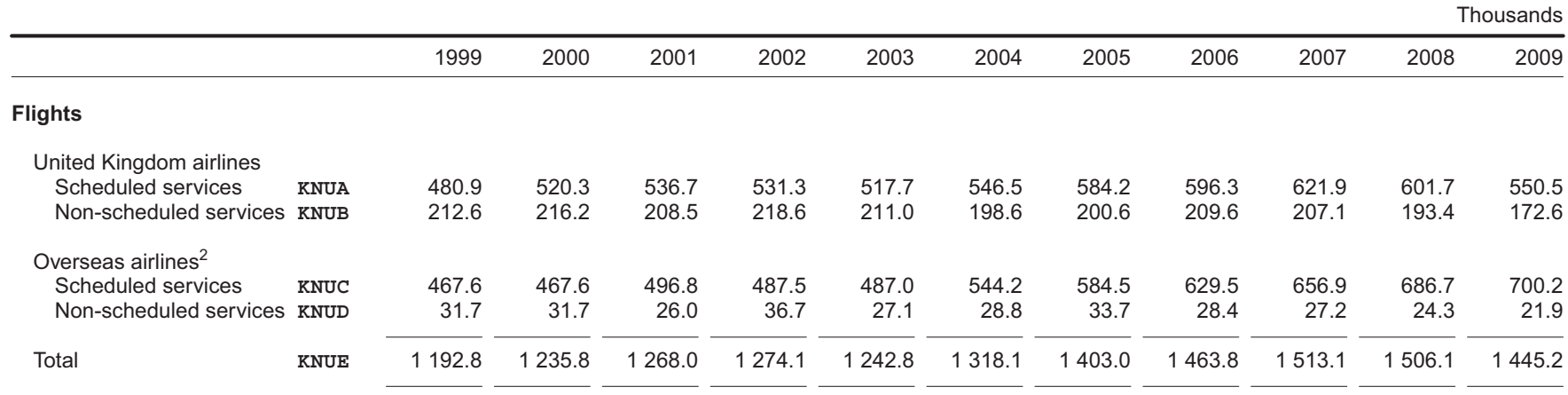

\section{Passengers carried}

United Kingdom airlines

$\begin{array}{llllllllllllllll}\text { Scheduled services } \quad \text { KNUF } \quad 50148.5 & 54522.8 & 53591.7 & 54360.0 & 56476.7 & 63216.1 & 69 & 106.2 & 72 & 196.4 & 76 & 959.9 & 76 & 636.5 & 71 & 586.9\end{array}$ Non-scheduled services KNUG $\quad 32603.8 \quad 33185.9 \quad 34009.1 \quad 33935.7 \quad 33385.6 \quad 32195.7 \quad 30179.4 \quad 29725.5 \quad 28524.0 \quad 25906.9 \quad 21774.2$

Overseas airlines ${ }^{2}$

$\begin{array}{lllllllllllllll}\text { Scheduled services } \quad \text { KNUH } \quad 46628.0 & 46627.9 & 51107.8 & 51317.6 & 54504.0 & 60278.0 & 67634.9 & 74670.8 & 79820.1 & 83 & 176.7 & 84 & 321.0\end{array}$

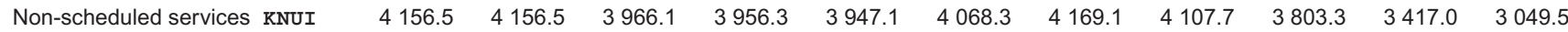

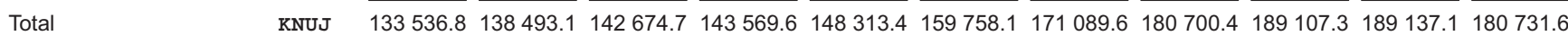

1 Excludes travel to and from the Channel Islands.

Source: Civil Aviation Authority: 02074536246

2 Includes airlines of overseas UK Territories. 


\begin{tabular}{|c|c|c|c|c|c|c|c|c|c|c|c|c|c|}
\hline & & & 1999 & 2000 & 2001 & 2002 & 2003 & 2004 & 2005 & 2006 & 2007 & 2008 & 2009 \\
\hline All services & & Unit & & & & & & & & & & & \\
\hline \multicolumn{14}{|l|}{ Aircraft stage flights: } \\
\hline Number & KNFA & Numbers & 835031 & 878582 & 921556 & 911518 & 895095 & 926498 & 1016354 & 1037729 & 1052799 & 1056298 & 1001504 \\
\hline Average length & KNFB & Kilometres & 1134.0 & 1156.0 & 1138.0 & 1149.0 & 1215.0 & 1227.0 & 1304.0 & 1349.0 & 1400.0 & 1427.9 & 1441.3 \\
\hline Aircraft-kilometres flown & KNFC & Millions & 947.0 & 1016.0 & 1049.0 & 1047.0 & 1088.0 & 1137.0 & 1325.0 & 1400.0 & 1474.0 & 1508.3 & 1443.5 \\
\hline Passengers uplifted & KNFD & " & 65.0 & 70.0 & 70.0 & 72.0 & 76.0 & 83.0 & 94.0 & 98.0 & 102.0 & 104.7 & 102.5 \\
\hline Cargo uplifted: ${ }^{2}$ & KNFF & Tonnes & 860291.0 & 897184.0 & 742705.0 & 768736.0 & 800645.0 & 842912.0 & 921412.0 & 946365.0 & 941421.0 & 979791.0 & 900668.0 \\
\hline Tonne-kilometres used: & & Millions & & & & & & & & & & & \\
\hline Passenger & $\mathrm{KNFH}$ & $"$ & 15518.0 & 16507.0 & 15258.0 & 15035.0 & 15419.0 & 15580.0 & 15044.0 & 16090.0 & 17246.0 & 17717.7 & 17474.4 \\
\hline Freight & KNFI & $"$ & 4925.0 & 5160.0 & 4548.0 & 4941.0 & 5187.0 & 5297.0 & 5998.0 & 6213.0 & 6199.0 & 6283.8 & 5863.7 \\
\hline Mail & KNFJ & $"$ & 153.0 & 179.0 & 102.0 & 57.0 & 55.0 & 75.0 & 90.0 & 99.0 & 112.0 & 99.0 & 88.6 \\
\hline Total & KNFG & $"$ & 20596.0 & 21846.0 & 19908.0 & 20032.0 & 20660.0 & 20952.0 & 21133.0 & 22402.0 & 23557.0 & 24100.5 & 23426.7 \\
\hline \multicolumn{14}{|l|}{ Domestic services } \\
\hline \multicolumn{14}{|l|}{ Aircraft stage flights: } \\
\hline Number & KNFK & Numbers & 354864 & 353525 & 365881 & 359400 & 345954 & 373858 & 394069 & 399438 & 383591 & 369499 & 341207 \\
\hline Average length & KNFL & Kilometres & 337.0 & 344.0 & 350.0 & 350.0 & 357.0 & 360.0 & 374.0 & 371.0 & 367.0 & 469.0 & 360.8 \\
\hline Aircraft-kilometres flown & KNFM & Millions & 120.0 & 121.0 & 128.0 & 126.0 & 123.0 & 135.0 & 147.0 & 148.0 & 140.0 & 173.3 & 123.1 \\
\hline Passengers uplifted & KNFN & " & 17.0 & 18.0 & 18.0 & 20.0 & 21.0 & 22.0 & 23.0 & 23.0 & 22.0 & 21.0 & 19.5 \\
\hline Seat-kilometres used & KNFO & $"$ & 7184.0 & 7542.0 & 7645.0 & 8322.0 & 8904.0 & 9263.0 & 9795.0 & 9800.0 & 9449.0 & 8951.5 & 8326.3 \\
\hline Cargo uplifted: ${ }^{2}$ & KNFP & Tonnes & 25964 & 24644 & 19498 & 16755 & 17248 & 14862 & 10015 & 8498 & 7099 & 6125 & 5202 \\
\hline Tonne-kilometres used: & & Millions & & & & & & & & & & & \\
\hline Passenger & KNFR & $"$ & 610.0 & 640.0 & 649.0 & 703.0 & 738.0 & 757.0 & 784.0 & 759.0 & 733.0 & 720.2 & 644.8 \\
\hline Freight & KNFS & $"$ & 6.0 & 6.0 & 4.0 & 4.0 & 3.0 & 3.0 & 3.0 & 2.0 & 2.0 & 1.8 & 1.6 \\
\hline Mail & KNFT & $"$ & 4.0 & 4.0 & 4.0 & 3.0 & 3.0 & 3.0 & - & 1.0 & 1.0 & 0.1 & 0.7 \\
\hline Total & KNFQ & $"$ & 620.0 & 650.0 & 656.0 & 709.0 & 744.0 & 762.0 & 787.0 & 762.0 & 735.0 & 722.1 & 647.1 \\
\hline
\end{tabular}

International services

Aircraft stage flights:

Number

$\begin{array}{llllllllllllll}480167 & 525057 & 555675 & 552118 & 549141 & 552640 & 622285 & 638291 & 669208 & 686799 & 660027\end{array}$ $\begin{array}{lllllllllll}1723.0 & 1704.0 & 1656.0 & 1670.0 & 1758.0 & 2148.0 & 1893.0 & 1960.0 & 1993.0 & 1994.4 & 2000.5\end{array}$

Aircraft-kilometres flown KNF

Passengers uplifted

Seat-kilometres used

82

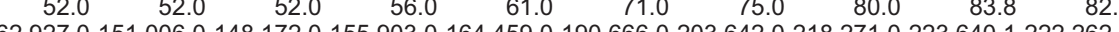

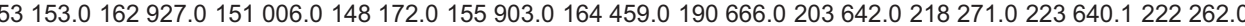

Cargo uplifted: ${ }^{2}$

KNFZ Tonnes

$834327872540 \quad 723206 \quad 751975$

Tonne-kilometres used:

Passenger

Freight

Millions

KNJX "

KNJY "

KNJZ

$14908.0 \quad 15867.0 \quad 14$

$\begin{array}{lllllllllll}919.0 & 5154.0 & 4544.0 & 4937.0 & 5184.0 & 5294.0 & 5995.0 & 6383.0 & 6197.0 & 6282.0 & 5862.1\end{array}$

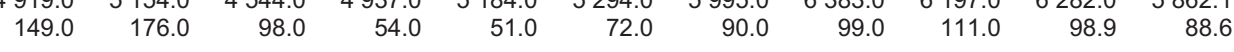

$\begin{array}{llllllllllll}19976.0 & 21 & 197.0 & 19252.0 & 19322.0 & 19916.0 & 20190.0 & 20345.0 & 21813.0 & 22822.0 & 23378.4 & 22780.3\end{array}$

Total

KNJW 
Thousands and tonnes

\begin{tabular}{|c|c|c|c|c|c|c|c|c|c|c|c|}
\hline & & 2000 & 2001 & 2002 & 2003 & 2004 & 2005 & 2006 & 2007 & 2008 & 2009 \\
\hline \multicolumn{12}{|c|}{ Movement of civil aircraft (thousands) } \\
\hline \multicolumn{12}{|l|}{ Commercial } \\
\hline Transport & KNQC & 2045 & 2095 & 2094 & 2160 & 2277 & 2406 & 2451 & 2494 & 2407 & 2195 \\
\hline Other $^{2}$ & KNQD & 159 & 150 & 120 & 117 & 116 & 120 & 129 & 124 & 113 & 96 \\
\hline Total & $\mathrm{KNQB}$ & 2204 & 2245 & 2214 & 2277 & 2393 & 2526 & 2580 & 2609 & 2520 & 2291 \\
\hline Total & KNQA & 3390 & 3452 & 3314 & 3463 & 3528 & 3655 & 3639 & 3637 & 3484 & 3203 \\
\hline \multicolumn{12}{|l|}{ Passengers handled } \\
\hline Terminal & KNQG & 179885 & 181231 & 188761 & 199950 & 215681 & 228214 & 235139 & 240722 & 235359 & 218126 \\
\hline Transit & $\mathrm{KNQH}$ & 1167 & 1087 & 1054 & 990 & 950 & 984 & 1016 & 963 & 735 & 519 \\
\hline \multicolumn{12}{|c|}{ Commercial freight handled ${ }^{4}$ (tonnes) } \\
\hline Set down & KNQJ & 1174635 & 1093142 & 1124026 & 1172552 & 1267411 & 1282724 & 1277177 & 1316359 & 1274539 & 1120886 \\
\hline Picked up & KNQK & 1139292 & 1052379 & 1071407 & 1035680 & 1103539 & 1080620 & 1038261 & 1009414 & 1007616 & 926974 \\
\hline Total & KNQI & 2313927 & 2145521 & 2195433 & 2208232 & 2370950 & 2363344 & 2315438 & 2325773 & 2282155 & 2047860 \\
\hline \multicolumn{12}{|l|}{ Mail handled } \\
\hline Set down & KNQM & 101743 & 98690 & 90738 & 86415 & 108481 & 102344 & 91535 & 102027 & 111002 & 96183 \\
\hline Picked up & KNQN & 123352 & 117389 & 99747 & 93096 & 112424 & 110576 & 98391 & 105755 & 123014 & 112001 \\
\hline Total & KNQL & 225095 & 216079 & 190485 & 179511 & 220905 & 212920 & 189926 & 207790 & 234016 & 208184 \\
\hline
\end{tabular}

1 Figures exclude Channel Island and Isle of Man Airports.

4 With effect from 2001, passengers, freight and mail handled exclude traffic car-

2 Local pleasure flights for reward (eg aerial survey work, crop dusting and ried on air taxi operations.

delivery of empty aircraft) and empty positioning flights.

3 Test and Training flights, Other flights by Air Transport Operators, Aero-club,

Private, Official, Military \& Business Aviation.

\section{Household digital television ${ }^{1}$ : by type of service Se $^{2,3}$}

\begin{tabular}{|c|c|c|c|c|}
\hline & Total digital television & Digital satellite & Digital terrestrial & Digital cable \\
\hline & IM6S & IM6T & IM6U & IM6V \\
\hline 2000 & 15.5 & 17.6 & 2.8 & 13.6 \\
\hline 2001 & 30.9 & 22.4 & 4.5 & 14.8 \\
\hline 2002 & 38.5 & 25.0 & 5.2 & 14.5 \\
\hline 2003 & 43.2 & 28.6 & 5.9 & 13.4 \\
\hline 2004 & 53.0 & 29.1 & 14.1 & 13.5 \\
\hline 2005 & 61.9 & 31.3 & 20.3 & 13.2 \\
\hline 2006 & 69.7 & 33.1 & 25.3 & 13.2 \\
\hline 2007 & 79.6 & 35.7 & 33.0 & 13.0 \\
\hline 2008 & 87.1 & 36.6 & 37.9 & 12.5 \\
\hline 2009 & 89.2 & 36.8 & 38.5 & 12.9 \\
\hline
\end{tabular}

1 Multichannel take-up on main sets.

Source: Ofcom: 02079813000

2 Data are at the end of the first quarter in each year.

3 GfK research from Q1 2007 onwards, previous years use platform operator

data, research and Ofcom estimates. 
Transport and communications

15.29 Telephons and The mentenat

\begin{tabular}{|c|c|c|c|c|c|c|c|c|}
\hline & \multicolumn{8}{|c|}{ Call Revenue (All Operators) } \\
\hline & \multicolumn{4}{|c|}{ Business call revenue (£s millions) } & \multicolumn{4}{|c|}{ Residential call revenue ( $£$ s millions) } \\
\hline & $\begin{array}{r}\text { UK Geographic } \\
\text { calls }\end{array}$ & $\begin{array}{r}\text { International } \\
\text { calls }\end{array}$ & Calls to mobiles & Other calls ${ }^{1}$ & $\begin{array}{r}\text { UK Geographic } \\
\text { calls }\end{array}$ & $\begin{array}{r}\text { International } \\
\text { calls }\end{array}$ & Calls to mobiles & Other calls $^{1}$ \\
\hline & IM6A & IM6B & IM6C & IM6D & IM6E & IM6F & IM6G & $\mathrm{IM} 6 \mathrm{H}$ \\
\hline 2007 & 581 & 256 & 670 & 256 & 863 & 308 & 966 & 897 \\
\hline 2008 Q3 & 122 & 60 & 148 & 52 & 215 & 74 & 231 & 196 \\
\hline Q4 & 115 & 61 & 140 & 52 & 220 & 73 & 227 & 199 \\
\hline 2009 Q1 & 120 & 62 & 149 & 53 & 221 & 71 & 216 & 183 \\
\hline Q2 & 115 & 60 & 142 & 54 & 220 & 69 & 217 & 201 \\
\hline \multirow[t]{3}{*}{ Q3 } & 112 & 58 & 141 & 52 & 223 & 70 & 218 & 193 \\
\hline & \multicolumn{8}{|c|}{ Call Volumes (All Operators) } \\
\hline & $\begin{array}{r}\text { UK Geographic } \\
\text { calls }\end{array}$ & $\begin{array}{r}\text { International } \\
\text { calls }\end{array}$ & Calls to mobiles & Other calls ${ }^{1}$ & $\begin{array}{r}\text { UK Geographic } \\
\text { calls }\end{array}$ & $\begin{array}{r}\text { International } \\
\text { calls }\end{array}$ & Calls to mobiles & Other calls $^{1}$ \\
\hline & IM6I & IM6J & IM6K & IM6L & IM6M & IM6N & IM6O & IM6P \\
\hline 2007 & 29442 & 2724 & 6829 & 11076 & 66651 & 3166 & 7536 & 33513 \\
\hline 2008 & 26145 & 2382 & 6512 & 9283 & 65747 & 3790 & 6765 & 23179 \\
\hline 2008 Q3 & 6489 & 589 & 1638 & 2344 & 15935 & 933 & 1662 & 5589 \\
\hline Q4 & 6148 & 560 & 1555 & 2219 & 16402 & 964 & 1604 & 5129 \\
\hline 2009 Q1 & 6239 & 564 & 1599 & 2220 & 16719 & 1001 & 1551 & 5014 \\
\hline Q2 & 5839 & 524 & 1516 & 2122 & 15849 & 1021 & 1550 & 4726 \\
\hline \multirow[t]{3}{*}{ Q3 } & 5755 & 490 & 1514 & 2137 & 15949 & 1057 & 1568 & 4711 \\
\hline & \multicolumn{5}{|c|}{ Selected lines with Carrier Pre-Selection (000's) } & \multicolumn{3}{|c|}{ Exchange line numbers (All operators) } \\
\hline & \multicolumn{3}{|c|}{ CPS lines $^{2}$} & \multicolumn{2}{|c|}{ WLR lines ${ }^{3}$} & Business (000's) & $\operatorname{Re}$ & dential (000's) \\
\hline 2007 & \multicolumn{3}{|c|}{24026} & \multicolumn{2}{|c|}{17406} & \multicolumn{2}{|l|}{11667} & 25996 \\
\hline 2008 & \multicolumn{3}{|r|}{.. } & \multicolumn{2}{|r|}{.. } & \multicolumn{2}{|l|}{10482} & 24099 \\
\hline 2008 Q3 & & & .. & & .. & 11059 & & 24118 \\
\hline Q4 & & & .. & & .. & 10482 & & 24099 \\
\hline 2009 Q1 & & & .. & & .. & 10622 & & 24285 \\
\hline Q2 & & & .. & & .. & 10001 & & 23147 \\
\hline Q3 & & & .. & & .. & 9896 & & 23284 \\
\hline
\end{tabular}

Data taken from the Telecommunications Market Data Update Q4 2007.

1 Includes freephone, special services, premium rate, directory enquiries and all other call types.

2 Allows usage of any service provide through a BT line.

3 Service which any other operator takes control of all connections made

through a telephone line and connects subscription fee from the subscribers. 
Transport and communications

Selected uses of the Internet, United Kingdom: by age, 2009

\begin{tabular}{|c|c|c|c|c|c|c|}
\hline & \\
\hline & $16-24$ & $25-44$ & $45-54$ & $55-64$ & 65 plus & All \\
\hline Sending/receiving emails & 94 & 92 & 88 & 86 & 82 & 90 \\
\hline Finding information about goods or services & 64 & 83 & 80 & 81 & 75 & 78 \\
\hline Internet banking & 50 & 61 & 55 & 48 & 43 & 54 \\
\hline Reading or downloading online news, magazines & 46 & 58 & 52 & 47 & 44 & 52 \\
\hline Playing or downloading games, images, films or music & 70 & 46 & 35 & 26 & 16 & 44 \\
\hline Listening to web radio or watching web TV & 53 & 46 & 35 & 34 & 25 & 42 \\
\hline Posting messages to chat sites, blogs etc & 71 & 45 & 25 & 19 &.. & 40 \\
\hline Consulting the Internet with the purpose of learning & 41 & 39 & 38 & 32 & 26 & 37 \\
\hline Looking for information - education, training, courses & 53 & 38 & 36 & 21 & 15 & 36 \\
\hline Downloading software & 46 & 39 & 31 & 25 & 25 & 36 \\
\hline
\end{tabular}

Internet purchases by adults, United Kingdom

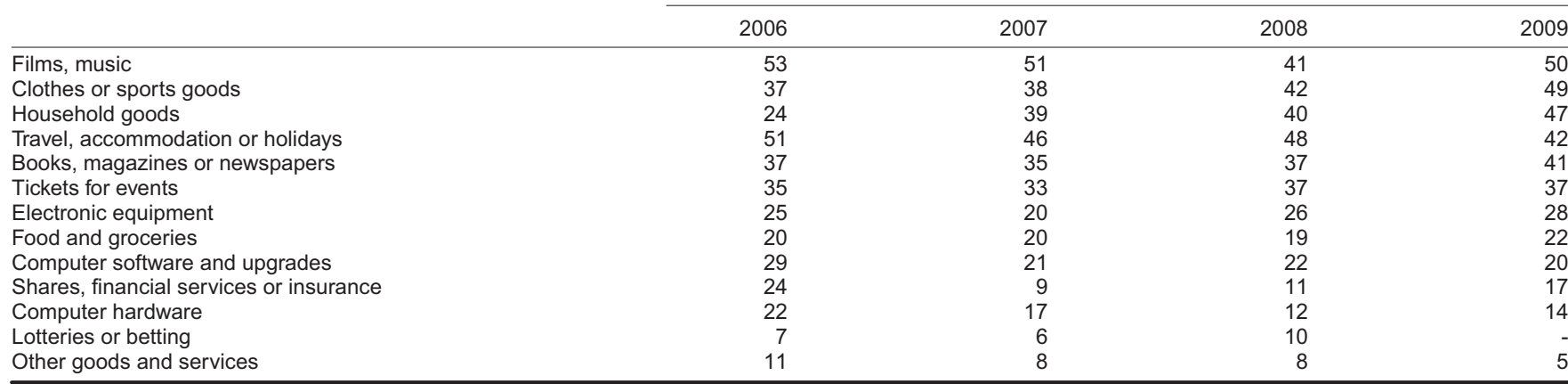

Other goods and services

Households with access to the Internet, Great Britain and United Kingdom, 2002 to $2009^{1}$

\begin{tabular}{|c|c|c|c|c|c|c|c|}
\hline \multicolumn{4}{|c|}{ Great Britain } & \multicolumn{4}{|c|}{ United Kingdom } \\
\hline 2002 & 46 & $11.02 \mathrm{~m}$ & - & 2002 & - & - & - \\
\hline 2003 & 50 & $11.88 \mathrm{~m}$ & 8 & 2003 & - & - & - \\
\hline 2004 & 51 & $12.16 \mathrm{~m}$ & 2 & 2004 & - & - & - \\
\hline 2007 & 61 & $14.94 \mathrm{~m}$ & 7 & 2007 & 61 & $15.23 \mathrm{~m}$ & 7 \\
\hline 2008 & 65 & $16.05 \mathrm{~m}$ & 7 & 2008 & 65 & $16.46 \mathrm{~m}$ & 8 \\
\hline 2008 & - & - & - & 2008 & 70 & 18.31 & 11 \\
\hline
\end{tabular}

1 The survey is conducted in the first quarter of each year but should not be confused with being quarterly figures as they relate to use at a point in time.

Sources: Office for National Statistics; Omnibus survey, Internet Access 2008; 


\begin{tabular}{|c|c|c|c|c|c|c|c|c|}
\hline & $\begin{array}{r}\text { Price of first } \\
\text { class stamp }(p)\end{array}$ & $\begin{array}{c}\text { Volume of first } \\
\text { class stamped } \\
\text { mail delivered } \\
\text { (million items) }\end{array}$ & $\begin{array}{r}\text { Total first } \\
\text { class mail } \\
\text { delivered } \\
\text { (million items) }\end{array}$ & $\begin{array}{l}\text { Price of second } \\
\text { class stamp (p) }\end{array}$ & $\begin{array}{r}\text { Volume of second } \\
\text { class stamped } \\
\text { mail delivered } \\
\text { (million items) }\end{array}$ & $\begin{array}{r}\text { Total second } \\
\text { class mail } \\
\text { delivered } \\
\text { (million items) }\end{array}$ & $\begin{array}{r}\text { Domestic parcels } \\
\text { (million) }\end{array}$ & $\begin{array}{r}\text { Internationa } \\
\text { parcels } \\
\text { (million) }\end{array}$ \\
\hline & IM7A & IM7B & IM7C & IM7D & IM7E & IM7F & IM7G & IM7H \\
\hline 2005 Q4 & 30 & 516 & 1499 & 21 & 536 & 2413 & 10.2 & 0.8 \\
\hline 2006 Q1 & 30 & 399 & 1280 & 21 & 284 & 2059 & 8.6 & 0.5 \\
\hline Q2 & 32 & 396 & 1292 & 23 & 267 & 1978 & 9.0 & 0.6 \\
\hline Q3 & 32 & 340 & 1217 & 23 & 242 & 1811 & 9.0 & 0.5 \\
\hline Q4 & 32 & 479 & 1377 & 23 & 507 & 2146 & 11.8 & 0.8 \\
\hline 2007 Q1 & 32 & 365 & 1189 & 23 & 272 & 1786 & 9.6 & 0.6 \\
\hline Q2 & 34 & 342 & 1185 & 24 & 244 & 1696 & 11.1 & 0.6 \\
\hline Q3 & 34 & 320 & 1118 & 24 & 229 & 1560 & 9.8 & 0.6 \\
\hline Q4 & 34 & 412 & 1250 & 24 & 457 & 1852 & 12.4 & 0.8 \\
\hline 2008 Q1 & 34 & 373 & 1195 & 24 & 282 & 1664 & 10.5 & 0.7 \\
\hline Q3 & 36 & 259 & 997 & 27 & 205 & 1362 & 9.7 & 0.9 \\
\hline Q4 & 36 & 351 & 1092 & 27 & 433 & 1580 & 12.1 & 1.0 \\
\hline 2009 Q1 & 36 & 295 & 974 & 27 & 250 & 1399 & 10.4 & 0.9 \\
\hline Q2 & 39 & 291 & 965 & 30 & 223 & 1285 & 11.5 & 0.3 \\
\hline Q3 & 39 & 232 & 856 & 30 & 183 & 1118 & 10.6 & 0.3 \\
\hline
\end{tabular}

\begin{tabular}{|c|c|c|c|c|c|c|c|c|c|c|c|c|}
\hline & & 1998 & 1999 & 2000 & 2001 & $2002^{3}$ & 2003 & 2004 & 2005 & 2006 & 2007 & $2008^{3}$ \\
\hline $\begin{array}{l}\text { Letters, etc posted (millions) } \\
\text { of which: }\end{array}$ & KMRA & 18350 & 18878 & 19711 & 20076 & 20648 & 21979 & 22837 & 24341 & 24880 & 24089 & 23705 \\
\hline Registered and insured & KMRB & 28.7 & 31.6 & 30.2 & 32.3 & 36.1 & 38.5 & 41.4 & 45.3 & 45.3 & 44.7 & 46.6 \\
\hline Airmail (Commonwealth and foreign) & KMRC & 658.4 & 693.2 & 672.3 & 659.2 & 600.7 & 541.6 & 512.0 & 457.9 & 502.2 & 541.0 & 470.3 \\
\hline Business reply and freepost items & KMRD & 524.7 & 503.6 & 475.3 & 487.4 & 486.2 & 434.4 & 397.7 & 401.1 & 402.3 & 373.7 & 349.1 \\
\hline \multicolumn{13}{|l|}{ Postal orders } \\
\hline Total issued (thousands) ${ }^{2}$ & KMRH & 31907 & 30289 & 30153 & 30931 & 29150 & 28666 & 28888 & 29344 & 20489 & 19714 & 16650 \\
\hline \multicolumn{13}{|l|}{ Television licences (thousands) } \\
\hline $\begin{array}{l}\text { In force on } 31 \text { March } \\
\text { of which: }\end{array}$ & KMQL & 21723 & 22240 & 22625 & 22839 & 23157 & 23486 & 23899 & 24162 & 24419 & 24546 & 24740 \\
\hline Colour & KMQM & 21344 & 21944 & 22413 & 22684 & 23040 & 23392 & 23824 & 24103 & 24370 & 24505 & 24706 \\
\hline
\end{tabular}

1 See chapter text.

2 Excluding those issued on HM ships, in many British possessions and in

Sources: Royal Mail Group : 02072502890

other places abroad. Up to 1998 includes Postal Orders issued Overseas Capita Business Services Limited: 0117302 1088;

and by Ministry of Defence. Post Office Limited: 02073207424

353 week year rather than 52 week standard 